\title{
Nutrient uptake and soil fertility status after harvest of Bt cotton as influenced by graded levels of NPK fertilizers in Alfisol
}

\author{
T. V. Jyothi* and N. S. Hebsur \\ Department of Soil Science and Agricultural Chemistry, UAS, Dharwad- 580005 (Karnataka), INDIA \\ *Corresponding author: E-mail: veeranna.jyothi@gmail.com
}

Received: March 11, 2017; Revised received: June 14, 2017; Accepted: October 25, 2017

\begin{abstract}
Field studies were conducted at farmer's fields in Jodalli (Kalghatgi taluk) and Pale (Hubballi taluk) villages in 2012-13 and 2013-14, respectively to investigate the effect of NPK fertilizers on uptake of nutrients by Bt cotton and soil fertility status at harvest in Alfisol. Among the different treatment combinations, the application of $150: 50: 75 \mathrm{~kg} \mathrm{~N}: \mathrm{P}_{2} \mathrm{O}_{5}: \mathrm{K}_{2} \mathrm{O}$ ha $^{-1}\left(\mathrm{~N}_{3} \mathrm{P}_{1} \mathrm{~K}_{2}\right)$ recorded significantly $(\mathrm{P}=0.05)$ higher nitrogen $\left(132.63 \mathrm{~kg}^{-1}\right)$, phosphorus $\left(31.26 \mathrm{~kg} \mathrm{ha}^{-1}\right)$ and potassium $\left(128.94 \mathrm{~kg} \mathrm{ha}^{-1}\right)$ uptake by cotton. The interaction effect with respect to total micronutrients $(\mathrm{Zn}, \mathrm{Fe}, \mathrm{Mn}$ and $\mathrm{Cu})$ uptake remained non significant at all the growth stages. Graded levels of fertilizers failed to exert significant impact on $\mathrm{pH}$ and electrical conductivity, soil organic carbon and available micronutrients during both the years of experimentation. The application of 100:50:50 kg N: $\mathrm{P}_{2} \mathrm{O}_{5}: \mathrm{K}_{2} \mathrm{O}$ ha ${ }^{-1}\left(\mathrm{~N}_{1} \mathrm{P}_{1} \mathrm{~K}_{1}\right)$ recorded significantly $(P=0.05)$ highest available nitrogen $\left(150.39 \mathrm{~kg} \mathrm{ha}^{-1}\right)$, available phosphorus $\left(37.98 \mathrm{~kg} \mathrm{ha}^{-1}\right)$ and available potassium $\left(230.99 \mathrm{~kg} \mathrm{ha}^{-1}\right)$ compared to rest of the treatments. The lowest available nitrogen (134.92 $\left.\mathrm{kg} \mathrm{ha}^{-1}\right)$, available phosphorus $\left(31.65 \mathrm{~kg} \mathrm{ha}^{-1}\right)$ and available potassium $\left(217.63 \mathrm{~kg} \mathrm{~h} \mathrm{a}^{-1}\right)$ were recorded in treatment receiving $150: 50: 75 \mathrm{~kg} \mathrm{~N}: \mathrm{P}_{2} \mathrm{O}_{5}: \mathrm{K}_{2} \mathrm{O}$ ha $^{-1}\left(\mathrm{~N}_{3} \mathrm{P}_{1} \mathrm{~K}_{2}\right)$.
\end{abstract}

Keywords: Alfisol, Cotton, Fertility, Fertilizers, Nutrient uptake

\section{INTRODUCTION}

Cotton (Gossypium species) enjoys a pre-eminent status among all the commercial crops in the country, being the principal raw material for flourishing textile industry. In India, in spite of several competitions from synthetic fibers in recent years, it is occupying a premiere position with 70 per cent share in the textile industry (Waiker et al., 2015). The nutrient management in cotton is a complex phenomenon due to its long duration and indeterminate growth habit where simultaneous production of vegetative and reproductive structures during the active growth phase takes place. Cotton plant being a heavy feeder, needs proper supply of plant nutrients for its successive cultivation (Tayade and Dhoble, 2010). Cotton removes good amounts of plant nutrients from the soil. The choice of appropriate nutrient management and crop rotation determines the efficiency of nutrients. Nutrient uptake by cotton is driven by the demand for nutrients from the developing crop, which is regulated by the supply of nutrients from the soil. Nutrients are taken up throughout the growing season and in proportion with the demand for nutrients as dictated by the developing crop biomass and boll load. The rates of nutrient uptake increase at flowering through fruiting, and then slow as the bolls mature (Mullins and Burmester, 2010).

Cotton has high demand for nutrients such as $\mathrm{N}$ and $\mathrm{K}$ in excess of $200 \mathrm{~kg} \mathrm{ha}^{-1}$ and daily accumulation rates of up to $4 \mathrm{~kg} \mathrm{ha}^{-1} \mathrm{day}^{-1}$, whereas trace nutrient needs are more easily met (up to $1 \mathrm{~kg} \mathrm{ha}^{-1}$ ) with fertilizer application where appropriate. Understandably, $\mathrm{N}$ has been more thoroughly studied than other nutrients, as $\mathrm{N}$ is most commonly deficient in agricultural systems without fertilizer addition. Redistribution of nutrients from vegetative to reproductive plant parts is a vital component of cotton plant nutrition, particularly for nitrogen and phosphorus (Rochester et al., 2012). In this direction, a detailed research was taken up with an objective of studying the effect of NPK fertilizers on nutrient uptake by Bt cotton and soil fertility status after harvest of cotton in Alfisol.

\section{MATERIALS AND METHODS}

Using factorial randomized complete block design with three replications and nineteen treatments we conducted two year field experiments in farmer's field one at Jodalli village (Kalghatgi taluk) in 2012-13 situated at $15^{\circ} 19^{\prime} 865^{\prime \prime}$ North latitude and $75^{\circ} 00^{\prime} 65^{\prime \prime}$ East longitude and another at Pale village (Hubballi taluk) in 2013-14 situated at $15^{0} 14^{\prime} 404^{\prime \prime}$ North latitude and $75^{\circ} 08^{\prime} 600^{\prime \prime}$ East longitude under protective irrigated condition with an aim to evaluate the effect of NPK levels on uptake of nutrients by Bt cotton and soil fertility status after harvest of the crop in Alfisol. The farmer of Jodalli village did not agree to take up the experiment during second year. Hence, the experiment was conducted at Pale. Composite soil samples were 
drawn from each experimental site before sowing and were analyzed for physico-chemical properties by following standard analytical procedures. The soil of experimental site was sandy loam in texture with acidic $\mathrm{pH}$ (6.27) and non-saline. The fertility status of the soil was low and medium in the available $\mathrm{N}, \mathrm{P}_{2} \mathrm{O}_{5}$, and $\mathrm{K}_{2} \mathrm{O}$ and the values were $131.40 \mathrm{~kg} \mathrm{ha}^{-1}, 28.60 \mathrm{~kg} \mathrm{ha}^{-1}$ and $208.90 \mathrm{~kg} \mathrm{ha}^{-1}$, respectively. The spacing adopted was $90 \mathrm{~cm}$ and between rows and $60 \mathrm{~cm}$ between plants for hybrid cotton. The treatment details are given below.

\section{Treatment details}

\section{A. Factor - I (N levels)}

$\mathrm{N}_{1}: 100 \mathrm{~kg} \mathrm{ha}^{-1}, \mathrm{~N}_{2}: 125 \mathrm{~kg} \mathrm{ha}^{-1}, \mathrm{~N}_{3}: 150 \mathrm{~kg} \mathrm{ha}^{-1}$

B. Factor - II ( $\mathbf{P}_{2} \mathrm{O}_{5}$ levels $)$

$\mathrm{P}_{1}: 50 \mathrm{~kg} \mathrm{ha}^{-1}, \mathrm{P}_{2}: 75 \mathrm{~kg} \mathrm{ha}^{-1}$

C. Factor - III ( $\mathrm{K}_{2} \mathrm{O}$ levels)

$\mathrm{K}_{1}: 50 \mathrm{~kg} \mathrm{ha}^{-1}, \mathrm{~K}_{2}: 75 \mathrm{~kg} \mathrm{ha}^{-1}, \mathrm{~K}_{3}: 100 \mathrm{~kg} \mathrm{ha}^{-1}$

Absolute control: Gap filling was done after seven days of sowing to avoid patchy crop stand. To maintain desired plant density, thinning was done at about 20 days after sowing (DAS). Entire recommended dose of phosphorus and potassium and 50 per cent of nitrogen were applied after germination by ring method. Remaining 50 per cent of nitrogen was applied at 60 DAS as per the package of practice, UAS, Dharwad. Adequate plant protection measures were taken as per the recommended package for $\mathrm{Bt}$ cotton as and when required at various growth stages commonly to all the treatments.

Treatment wise plant samples were collected at 60, 90, 120 DAS and harvest by uprooting the entire plant carefully. At 120 DAS and harvest stage, the cotton was picked separately and then the plant was uprooted. The plant samples were first washed with distilled water and dried in shade and then oven dried at $60{ }^{\circ} \mathrm{C}$ to get constant weight. Then, the plant samples were powdered in a grinder and stored in butter paper bags. Plant samples were digested with diacid mixture (nitric acid and perchloric acid in 9:4 ratio), after predigesting with concentrated nitric acid until clear and white residue was obtained. Then, the residue was dissolved in $6 \mathrm{~N} \mathrm{HCl}$ and finally volume was made up to $100 \mathrm{ml}$ using distilled water (Tandon, 1998).

Total nitrogen was determined by micro Kjeldahl's method using digestion mixture consisting of $\mathrm{K}_{2} \mathrm{SO}_{4}$, $\mathrm{CuSO}_{4}$ and selenium powder in presence of $\mathrm{H}_{2} \mathrm{SO}_{4}$. Half a gram of plant sample was digested in a block digestion unit. After complete digestion, the samples were distilled using micro Kjeldahl unit and the liberated ammonia was trapped in boric acid containing mixed indicator and titrated against $0.01 \mathrm{~N} \mathrm{H}_{2} \mathrm{SO}_{4}$ (Tandon, 1998). The phosphorus in the plant digest was determined by vanadomolybdate yellow colour method in nitric acid medium. The intensity of colour was read at $420 \mathrm{~nm}$ wavelength using spectrophotometer (Tandon, 1998). Potassium in the plant digest was estimated by automizing the diluted plant extract in the flame photometer (Tandon, 1998). Micronutrients in the plant digest were estimated by atomic absorption spectrophotometer (Tandon, 1998).

The soil $\mathrm{pH}$ was measured in 1:2.5 soil water suspension using $\mathrm{pH}$ meter and $\mathrm{EC}\left(\mathrm{dS} \mathrm{m}{ }^{-1}\right)$ was measured in the supernatant solution of 1:2.5 soil water extract using conductivity bridge (Sparks, 1996). Organic carbon was estimated by Walkley and Black's wet oxidation method (Sparks, 1996). Available nitrogen was estimated by modified alkaline $\mathrm{KMnO}_{4}$ method (Sharawat and Burford, 1982). Available phosphorus was extracted with Olsen's and Bray's reagent depending on their $\mathrm{pH}$ and the amount of $\mathrm{P}$ in the extract was estimated by chlorostannous reduced phosphomolybdate blue colour method using spectrophotometer at wavelength of $660 \mathrm{~nm}$ (Sparks, 1996). Available potassium was extracted with neutral normal ammonium acetate extract and determined by using flame photometer as described by Sparks (1996). The micronutrients were extracted with DTPA extractant and the aliquot was assessed by using Atomic Absorption Spectrophotometer (Lindsay and Norvell, 1978).

\section{RESULTS AND DISCUSSION}

Effect of NPK fertilizers on nutrient uptake by Bt cotton in Alfisols: The uptake of NPK nutrients increased significantly $(\mathrm{P}=0.05)$ with increase in levels of nitrogen, phosphorus and potassium at all the growth stages except at 60 DAS. Among the different treatment combinations, the application of 150:50:75 $\mathrm{kg} \mathrm{N}: \mathrm{P}_{2} \mathrm{O}_{5}: \mathrm{K}_{2} \mathrm{O}$ ha $^{-1}\left(\mathrm{~N}_{3} \mathrm{P}_{1} \mathrm{~K}_{2}\right)$ recorded significantly $(\mathrm{P}=0.05)$ the highest uptake of nitrogen $(132.63 \mathrm{~kg} \mathrm{ha}$ $\left.{ }^{1}\right)$, phosphorus $\left(31.26 \mathrm{~kg} \mathrm{ha}^{-1}\right)$ and potassium (128.94 $\mathrm{kg} \mathrm{ha}^{-1}$ ) over rest of the treatments at harvest. However, the treatment $\mathrm{N}_{3} \mathrm{P}_{1} \mathrm{~K}_{3} \quad(150: 50: 100 \quad \mathrm{~kg}$ $\mathrm{N}: \mathrm{P}_{2} \mathrm{O}_{5}: \mathrm{K}_{2} \mathrm{O}$ ha ${ }^{-1}$ ) was on par with $\mathrm{N}_{3} \mathrm{P}_{1} \mathrm{~K}_{2}$ at all the growth stages (Table 1 to 6 ). The interaction effect with respect to total micronutrients $(\mathrm{Zn}, \mathrm{Fe}, \mathrm{Mn}$ and $\mathrm{Cu})$ uptake remained non significant at all the growth stages. However, the treatment receiving 150:50:75 kg $\mathrm{N}: \mathrm{P}_{2} \mathrm{O}_{5}: \mathrm{K}_{2} \mathrm{O}$ ha ${ }^{-1}\left(\mathrm{~N}_{3} \mathrm{P}_{1} \mathrm{~K}_{2}\right)$ recorded higher total micronutrients $(\mathrm{Zn}, \mathrm{Fe}, \mathrm{Mn}$ and $\mathrm{Cu})$ uptake at all the growth stages. The higher uptake could be attributed to the highest yield observed in the treatment. Normally uptake follows the dry matter yield and yield of crops. In a study conducted by Zakaria et al. (2009) also obtained similar findings in which $\mathrm{N} @ 142.8 \mathrm{~kg} \mathrm{~N} \mathrm{ha}^{-1}$ and $\mathrm{K}_{2} \mathrm{O} @ 1.15 \mathrm{~kg} \mathrm{ha}^{-1}$ recorded $1560.8 \mathrm{mg} \mathrm{plant}^{-1}$ and $1615 \mathrm{mg}$ plant $^{-1}$, respectively as compared to rest of the treatments in Egyptian cotton. A synergism in the uptake of $\mathrm{N}, \mathrm{P}$ and $\mathrm{K}$ occurs as $\mathrm{K}$ is the main counter ion for root nitrate uptake and xylem transport to shoots (Marschner, 1995), i.e. increasing $\mathrm{N}$ and $\mathrm{P}$ uptake rates require increasing uptake of $\mathrm{K}$ for balancing charges (Peuke et al., 2002). These findings reveal the fact that $\mathrm{N}$ is an essential nutrient in building the plant dry matter as well as many energy-rich com- 
T.V. Jyothi and N.S. Hebsur / J. Appl. \& Nat. Sci. 9 (4): 2315 - 2326 (2017)

Table 1: Nitrogen uptake by Bt cotton as influenced by different levels of NPK at 60 and 90 DAS in Alfisol.

\begin{tabular}{|c|c|c|c|c|c|c|}
\hline \multirow{3}{*}{ Treatments } & \multicolumn{6}{|c|}{ Nitrogen uptake $\left(\mathrm{kg} \mathrm{ha}^{-1}\right)$} \\
\hline & \multicolumn{3}{|c|}{ 60 DAS } & \multicolumn{3}{|c|}{ 90 DAS } \\
\hline & $2012-13$ & 2013-14 & Pooled & $2012-13$ & 2013-14 & Pooled \\
\hline $\mathrm{N}_{1}$ & 25.09 & 26.40 & 25.74 & 65.57 & $\begin{array}{l}67.29 \\
\end{array}$ & 66.43 \\
\hline $\mathrm{N}_{2}$ & 27.40 & 28.71 & 28.05 & 71.73 & 73.29 & 72.51 \\
\hline $\mathrm{N}_{3}$ & 28.72 & 30.22 & 29.47 & 76.76 & 79.25 & 78.00 \\
\hline S.Em.土 & 0.25 & 0.31 & 0.19 & 0.42 & 0.37 & 0.27 \\
\hline C.D. at 5\% & 0.71 & 0.91 & 0.56 & 1.21 & 1.07 & 0.77 \\
\hline $\mathrm{P}_{1}$ & 26.79 & 28.44 & 27.61 & 70.76 & 72.94 & 71.85 \\
\hline $\mathrm{P}_{2}$ & 27.35 & 28.44 & 27.89 & 71.94 & 73.62 & 72.78 \\
\hline S.Em. \pm & 0.20 & 0.26 & 0.16 & 0.34 & 0.30 & 0.22 \\
\hline C.D. at 5\% & NS & NS & NS & 0.99 & NS & 0.63 \\
\hline $\mathrm{K}_{1}$ & 26.66 & 27.75 & 27.21 & 70.39 & 71.78 & 71.09 \\
\hline $\mathrm{K}_{2}$ & 27.16 & 28.40 & 27.78 & 71.60 & 73.69 & 72.64 \\
\hline $\mathrm{K}_{3}$ & 27.38 & 29.18 & 28.28 & 72.07 & 74.37 & 73.22 \\
\hline S.Em. \pm & 0.25 & 0.31 & 0.19 & 0.42 & 0.37 & 0.27 \\
\hline C.D. at 5\% & NS & 0.91 & 0.56 & 1.21 & 1.07 & 0.77 \\
\hline $\mathrm{N}_{1} \mathrm{P}_{1}$ & 24.47 & 25.83 & 25.15 & 65.11 & 67.24 & 66.17 \\
\hline $\mathrm{N}_{1} \mathrm{P}_{2}$ & 25.70 & 26.97 & 26.33 & 66.03 & 67.35 & 66.69 \\
\hline $\mathrm{N}_{2} \mathrm{P}_{1}$ & 26.07 & 27.78 & 26.92 & 69.68 & 71.57 & 70.63 \\
\hline $\mathrm{N}_{2} \mathrm{P}_{2}$ & 28.74 & 29.63 & 29.19 & 73.78 & 75.01 & 74.40 \\
\hline $\mathrm{N}_{3} \mathrm{P}_{1}$ & 29.84 & 31.71 & 30.77 & 77.49 & 80.01 & 78.75 \\
\hline $\mathrm{N}_{3} \mathrm{P}_{2}$ & 27.60 & 28.73 & 28.16 & 76.02 & 78.49 & 77.26 \\
\hline S.Em. . & 0.35 & 0.45 & 0.27 & 0.59 & 0.53 & 0.38 \\
\hline C.D. at 5\% & 1.00 & 1.28 & 0.79 & 1.71 & 1.52 & 1.09 \\
\hline $\mathrm{N}_{1} \mathrm{~K}_{1}$ & 24.13 & 25.27 & 24.70 & 64.25 & 65.14 & 64.69 \\
\hline $\mathrm{N}_{1} \mathrm{~K}_{2}$ & 25.09 & 26.09 & 25.59 & 65.61 & 67.15 & 66.38 \\
\hline $\mathrm{N}_{1} \mathrm{~K}_{3}$ & 26.03 & 27.84 & 26.93 & 66.85 & 69.59 & 68.22 \\
\hline $\mathrm{N}_{2} \mathrm{~K}_{1}$ & 26.80 & 27.76 & 27.28 & 70.10 & 71.08 & 70.59 \\
\hline $\mathrm{N}_{2} \mathrm{~K}_{2}$ & 27.17 & 28.38 & 27.78 & 72.01 & 73.54 & 72.78 \\
\hline $\mathrm{N}_{2} \mathrm{~K}_{3}$ & 28.23 & 29.98 & 29.11 & 73.08 & 75.25 & 74.17 \\
\hline $\mathrm{N}_{3} \mathrm{~K}_{1}$ & 29.06 & 30.21 & 29.63 & 76.83 & 79.13 & 77.98 \\
\hline $\mathrm{N}_{3} \mathrm{~K}_{2}$ & 29.21 & 30.73 & 29.97 & 77.17 & 80.36 & 78.77 \\
\hline $\mathrm{N}_{3} \mathrm{~K}_{3}$ & 27.88 & 29.71 & 28.79 & 76.27 & 78.26 & 77.27 \\
\hline S.Em. \pm & 0.43 & 0.55 & 0.34 & 0.73 & 0.65 & 0.46 \\
\hline C.D. at $5 \%$ & 1.23 & 1.57 & 0.97 & NS & 1.86 & 1.34 \\
\hline $\mathrm{P}_{1} \mathrm{~K}_{1}$ & 26.20 & 27.50 & 26.85 & 69.93 & 71.50 & 70.71 \\
\hline $\mathrm{P}_{1} \mathrm{~K}_{2}$ & 26.88 & 28.74 & 27.81 & 71.26 & 73.53 & 72.39 \\
\hline $\mathrm{P}_{1} \mathrm{~K}_{3}$ & 27.29 & 29.08 & 28.19 & 71.10 & 73.79 & 72.45 \\
\hline $\mathrm{P}_{2} \mathrm{~K}_{1}$ & 27.13 & 28.00 & 27.57 & 70.86 & 72.07 & 71.46 \\
\hline $\mathrm{P}_{2} \mathrm{~K}_{2}$ & 27.44 & 28.06 & 27.75 & 71.94 & 73.84 & 72.89 \\
\hline $\mathrm{P}_{2} \mathrm{~K}_{3}$ & 27.47 & 29.27 & 28.37 & 73.03 & 74.95 & 73.99 \\
\hline S.Em.土 & 0.35 & 0.45 & 0.27 & 0.59 & 0.53 & 0.38 \\
\hline C.D. at 5\% & NS & NS & NS & NS & NS & NS \\
\hline $\mathrm{N}_{1} \mathrm{P}_{1} \mathrm{~K}_{1}$ & 23.35 & 25.05 & 24.20 & 63.96 & 64.92 & 64.44 \\
\hline $\mathrm{N}_{1} \mathrm{P}_{1} \mathrm{~K}_{2}$ & 24.33 & 25.77 & 25.05 & 65.21 & 67.03 & 66.12 \\
\hline $\mathrm{N}_{1} \mathrm{P}_{1} \mathrm{~K}_{3}$ & 25.74 & 26.67 & 26.20 & 66.16 & 69.77 & 67.96 \\
\hline $\mathrm{N}_{1} \mathrm{P}_{2} \mathrm{~K}_{1}$ & 24.92 & 25.49 & 25.21 & 64.53 & 65.36 & 64.95 \\
\hline $\mathrm{N}_{1} \mathrm{P}_{2} \mathrm{~K}_{2}$ & 25.85 & 26.40 & 26.13 & 66.01 & 67.27 & 66.64 \\
\hline $\mathrm{N}_{1} \mathrm{P}_{2} \mathrm{~K}_{3}$ & 26.33 & 29.01 & 27.67 & 67.54 & 69.42 & 68.48 \\
\hline $\mathrm{N}_{2} \mathrm{P}_{1} \mathrm{~K}_{1}$ & 25.39 & 26.64 & 26.02 & 69.02 & 70.42 & 69.72 \\
\hline $\mathrm{N}_{2} \mathrm{P}_{1} \mathrm{~K}_{2}$ & 25.67 & 27.21 & 26.44 & 69.84 & 71.57 & 70.71 \\
\hline $\mathrm{N}_{2} \mathrm{P}_{1} \mathrm{~K}_{3}$ & 27.13 & 29.48 & 28.31 & 70.19 & 72.72 & 71.46 \\
\hline $\mathrm{N}_{2} \mathrm{P}_{2} \mathrm{~K}_{1}$ & 28.22 & 28.87 & 28.55 & 71.18 & 71.74 & 71.46 \\
\hline $\mathrm{N}_{2} \mathrm{P}_{2} \mathrm{~K}_{2}$ & 28.67 & 29.55 & 29.11 & 74.19 & 75.52 & 74.85 \\
\hline $\mathrm{N}_{2} \mathrm{P}_{2} \mathrm{~K}_{3}$ & 29.33 & 30.48 & 29.90 & 75.98 & 77.77 & 76.87 \\
\hline $\mathrm{N}_{3} \mathrm{P}_{1} \mathrm{~K}_{1}$ & 29.85 & 30.79 & 30.32 & 76.80 & 79.16 & 77.98 \\
\hline $\mathrm{N}_{3} \mathrm{P}_{1} \mathrm{~K}_{2}$ & 30.64 & 33.25 & 31.94 & 78.72 & 81.99 & 80.36 \\
\hline $\mathrm{N}_{3} \mathrm{P}_{1} \mathrm{~K}_{3}$ & 29.01 & 31.09 & 30.05 & 76.95 & 78.88 & 77.92 \\
\hline $\mathrm{N}_{3} \mathrm{P}_{2} \mathrm{~K}_{1}$ & 28.26 & 29.63 & 28.95 & 76.85 & 79.10 & 77.98 \\
\hline $\mathrm{N}_{3} \mathrm{P}_{2} \mathrm{~K}_{2}$ & 27.78 & 28.22 & 28.00 & 75.63 & 78.73 & 77.18 \\
\hline $\mathrm{N}_{3} \mathrm{P}_{2} \mathrm{~K}_{3}$ & 26.75 & 28.33 & 27.54 & 75.59 & 77.64 & 76.61 \\
\hline S.Em. \pm & 0.60 & 0.77 & 0.48 & 1.03 & 0.91 & 0.66 \\
\hline C.D. at 5\% & NS & NS & NS & 2.96 & 2.63 & 1.89 \\
\hline Control & 19.72 & 21.59 & 20.66 & 55.58 & 60.56 & 58.07 \\
\hline S.Em. \pm & 0.65 & 0.76 & 0.48 & 1.06 & 0.95 & 0.66 \\
\hline C.D. at 5\% & 1.87 & 2.17 & 1.37 & 3.04 & 2.73 & 1.88 \\
\hline
\end{tabular}

FYM $-5 \mathrm{t} \mathrm{ha}^{-1} ; \mathrm{N}_{1}-100 \mathrm{~kg} \mathrm{ha}^{-1} ; \mathrm{N}_{2}-125 \mathrm{~kg} \mathrm{ha}^{-1} ; \mathrm{N}_{3}-150 \mathrm{~kg} \mathrm{ha}^{-1} ; \mathrm{P}_{1}-50 \mathrm{~kg} \mathrm{ha}^{-1} ; \mathrm{P}_{2}-75 \mathrm{~kg} \mathrm{ha}^{-1} ; \mathrm{K}_{1}-50 \mathrm{~kg} \mathrm{ha}^{-1} ; \mathrm{K}_{2}-75 \mathrm{~kg} \mathrm{ha}^{-1} ;$ $\mathrm{K}_{3}-100 \mathrm{~kg} \mathrm{ha}^{-1}$; NS - Non significant ; DAS - Days after sowing 
T.V. Jyothi and N.S. Hebsur / J. Appl. \& Nat. Sci. 9 (4): 2315 - 2326 (2017)

Table 2. Nitrogen uptake by Bt cotton as influenced by different levels of NPK at 120 DAS and harvest in Alfisol.

\begin{tabular}{|c|c|c|c|c|c|c|}
\hline \multirow{3}{*}{ Treatments } & \multicolumn{6}{|c|}{ Nitrogen uptake $\left(\mathrm{kg} \mathrm{ha}^{-1}\right)$} \\
\hline & \multicolumn{3}{|c|}{120 DAS } & \multicolumn{3}{|c|}{ At harvest } \\
\hline & 2012-13 & 2013-14 & Pooled & 2012-13 & 2013-14 & Pooled \\
\hline $\mathrm{N}_{1}$ & 117.98 & 119.76 & 118.87 & 119.43 & 121.11 & 120.27 \\
\hline $\mathrm{N}_{2}$ & 121.22 & 124.00 & 122.61 & 123.88 & 125.25 & 124.56 \\
\hline $\mathrm{N}_{3}$ & 126.33 & 130.16 & 128.25 & 128.59 & 130.07 & 129.33 \\
\hline S.Em. \pm & 0.51 & 0.40 & 0.30 & 0.44 & 0.39 & 0.32 \\
\hline C.D. at $5 \%$ & 1.47 & 1.14 & 0.88 & 1.26 & 1.11 & 0.91 \\
\hline$P_{1}$ & 121.52 & 123.77 & 122.65 & 123.87 & 125.27 & 124.57 \\
\hline $\mathrm{P}_{2}$ & 122.16 & 125.51 & 123.84 & 124.06 & 125.69 & 124.88 \\
\hline S.Em. \pm & 0.42 & 0.32 & 0.25 & 0.36 & 0.32 & 0.26 \\
\hline C.D. at $5 \%$ & NS & 0.93 & 0.72 & NS & NS & NS \\
\hline $\mathrm{K}_{1}$ & 120.73 & 123.29 & 122.01 & 122.46 & 123.68 & 123.07 \\
\hline $\mathrm{K}_{2}$ & 122.29 & 125.07 & 123.68 & 124.07 & 125.88 & 124.98 \\
\hline $\mathrm{K}_{3}$ & 122.51 & 125.56 & 124.03 & 125.37 & 126.88 & 126.12 \\
\hline S.Em. \pm & 0.51 & 0.40 & 0.30 & 0.44 & 0.39 & 0.32 \\
\hline C.D. at $5 \%$ & 1.47 & 1.14 & 0.88 & 1.26 & 1.11 & 0.91 \\
\hline $\mathrm{N}_{1} \mathrm{P}_{1}$ & 117.36 & 119.23 & 118.30 & 118.91 & 120.77 & 119.84 \\
\hline $\mathrm{N}_{1} \mathrm{P}_{2}$ & 118.59 & 120.29 & 119.44 & 119.95 & 121.46 & 120.70 \\
\hline $\mathrm{N}_{2} \mathrm{P}_{1}$ & 119.08 & 120.74 & 119.91 & 122.16 & 123.48 & 122.82 \\
\hline $\mathrm{N}_{2} \mathrm{P}_{2}$ & 123.37 & 127.27 & 125.32 & 125.59 & 127.03 & 126.31 \\
\hline $\mathrm{N}_{3} \mathrm{P}_{1}$ & 128.13 & 131.36 & 129.74 & 130.53 & 131.56 & 131.04 \\
\hline $\mathrm{N}_{3} \mathrm{P}_{2}$ & 124.53 & 128.97 & 126.75 & 126.65 & 128.59 & 127.62 \\
\hline S.Em. \pm & 0.72 & 0.56 & 0.43 & 0.62 & 0.55 & 0.45 \\
\hline C.D. at $5 \%$ & 2.08 & 1.61 & 1.24 & 1.78 & 1.57 & 1.29 \\
\hline $\mathrm{N}_{1} \mathrm{~K}_{1}$ & 116.55 & 117.96 & 117.26 & 118.36 & 119.33 & 118.85 \\
\hline $\mathrm{N}_{1} \mathrm{~K}_{2}$ & 118.14 & 119.81 & 118.98 & 119.38 & 121.20 & 120.29 \\
\hline $\mathrm{N}_{1} \mathrm{~K}_{3}$ & 119.24 & 121.51 & 120.37 & 120.54 & 122.81 & 121.67 \\
\hline $\mathrm{N}_{2} \mathrm{~K}_{1}$ & 119.38 & 121.86 & 120.62 & 120.63 & 121.88 & 121.25 \\
\hline $\mathrm{N}_{2} \mathrm{~K}_{2}$ & 121.36 & 124.37 & 122.86 & 123.57 & 125.37 & 124.47 \\
\hline $\mathrm{N}_{2} \mathrm{~K}_{3}$ & 122.92 & 125.78 & 124.35 & 127.43 & 128.51 & 127.97 \\
\hline $\mathrm{N}_{3} \mathrm{~K}_{1}$ & 126.26 & 130.06 & 128.16 & 128.38 & 129.82 & 129.10 \\
\hline $\mathrm{N}_{3} \mathrm{~K}_{2}$ & 127.37 & 131.03 & 129.20 & 129.25 & 131.08 & 130.16 \\
\hline $\mathrm{N}_{3} \mathrm{~K}_{3}$ & 125.36 & 129.39 & 127.37 & 128.13 & 129.32 & 128.72 \\
\hline S.Em. \pm & 0.88 & 0.69 & 0.53 & 0.76 & 0.67 & 0.55 \\
\hline C.D. at $5 \%$ & NS & 1.97 & 1.52 & 2.18 & 1.93 & 1.58 \\
\hline $\mathrm{P}_{1} \mathrm{~K}_{1}$ & 120.20 & 122.11 & 121.15 & 122.38 & 123.35 & 122.86 \\
\hline $\mathrm{P}_{1} \mathrm{~K}_{2}$ & 122.08 & 124.50 & 123.29 & 124.40 & 126.22 & 125.31 \\
\hline $\mathrm{P}_{1} \mathrm{~K}_{3}$ & 122.30 & 124.72 & 123.51 & 124.82 & 126.24 & 125.53 \\
\hline $\mathrm{P}_{2} \mathrm{~K}_{1}$ & 121.27 & 124.48 & 122.87 & 122.54 & 124.01 & 123.27 \\
\hline $\mathrm{P}_{2} \mathrm{~K}_{2}$ & 122.50 & 125.64 & 124.07 & 123.74 & 125.55 & 124.64 \\
\hline $\mathrm{P}_{2} \mathrm{~K}_{3}$ & 122.72 & 126.40 & 124.56 & 125.91 & 127.51 & 126.71 \\
\hline S.Em. \pm & 0.72 & 0.56 & 0.43 & 0.62 & 0.55 & 0.45 \\
\hline C.D. at $5 \%$ & NS & NS & NS & NS & NS & NS \\
\hline $\mathrm{N}_{1} \mathrm{P}_{1} \mathrm{~K}_{1}$ & 115.77 & 117.17 & 116.47 & 117.75 & 119.48 & 118.61 \\
\hline $\mathrm{N}_{1} \mathrm{P}_{1} \mathrm{~K}_{2}$ & 117.74 & 119.20 & 118.47 & 118.94 & 120.55 & 119.74 \\
\hline $\mathrm{N}_{1} \mathrm{P}_{1} \mathrm{~K}_{3}$ & 118.58 & 121.32 & 119.95 & 120.05 & 122.28 & 121.16 \\
\hline $\mathrm{N}_{1} \mathrm{P}_{2} \mathrm{~K}_{1}$ & 117.34 & 118.75 & 118.04 & 118.98 & 119.19 & 119.08 \\
\hline $\mathrm{N}_{1} \mathrm{P}_{2} \mathrm{~K}_{2}$ & 118.53 & 120.42 & 119.48 & 119.83 & 121.85 & 120.84 \\
\hline $\mathrm{N}_{1} \mathrm{P}_{2} \mathrm{~K}_{3}$ & 119.90 & 121.69 & 120.79 & 121.04 & 123.33 & 122.18 \\
\hline $\mathrm{N}_{2} \mathrm{P}_{1} \mathrm{~K}_{1}$ & 117.39 & 118.77 & 118.08 & 119.67 & 120.12 & 119.90 \\
\hline $\mathrm{N}_{2} \mathrm{P}_{1} \mathrm{~K}_{2}$ & 119.09 & 121.43 & 120.26 & 122.55 & 124.56 & 123.55 \\
\hline $\mathrm{N}_{2} \mathrm{P}_{1} \mathrm{~K}_{3}$ & 120.75 & 122.01 & 121.38 & 124.28 & 125.76 & 125.02 \\
\hline $\mathrm{N}_{2} \mathrm{P}_{2} \mathrm{~K}_{1}$ & 121.37 & 124.94 & 123.15 & 121.59 & 123.64 & 122.61 \\
\hline $\mathrm{N}_{2} \mathrm{P}_{2} \mathrm{~K}_{2}$ & 123.63 & 127.30 & 125.47 & 124.60 & 126.18 & 125.39 \\
\hline $\mathrm{N}_{2} \mathrm{P}_{2} \mathrm{~K}_{3}$ & 125.10 & 129.55 & 127.33 & 130.59 & 131.26 & 130.92 \\
\hline $\mathrm{N}_{3} \mathrm{P}_{1} \mathrm{~K}_{1}$ & 127.42 & 130.38 & 128.90 & 129.73 & 130.44 & 130.08 \\
\hline $\mathrm{N}_{3} \mathrm{P}_{1} \mathrm{~K}_{2}$ & 129.40 & 132.86 & 131.13 & 131.72 & 133.55 & 132.63 \\
\hline $\mathrm{N}_{3} \mathrm{P}_{1} \mathrm{~K}_{3}$ & 127.56 & 130.82 & 129.19 & 130.15 & 130.69 & 130.42 \\
\hline $\mathrm{N}_{3} \mathrm{P}_{2} \mathrm{~K}_{1}$ & 125.11 & 129.74 & 127.43 & 127.04 & 129.20 & 128.12 \\
\hline $\mathrm{N}_{3} \mathrm{P}_{2} \mathrm{~K}_{2}$ & 125.34 & 129.21 & 127.27 & 126.79 & 128.61 & 127.70 \\
\hline $\mathrm{N}_{3} \mathrm{P}_{2} \mathrm{~K}_{3}$ & 123.15 & 127.96 & 125.56 & 126.11 & 127.95 & 127.03 \\
\hline S.Em. \pm & 1.25 & 0.97 & 0.75 & 1.07 & 0.95 & 0.78 \\
\hline C.D. at $5 \%$ & 3.60 & 2.79 & 2.15 & 3.08 & 2.73 & 2.24 \\
\hline Control & 110.93 & 113.88 & 112.41 & 113.30 & 115.72 & 114.51 \\
\hline S.Em. \pm & 1.37 & 1.27 & 0.78 & 1.05 & 1.02 & 0.78 \\
\hline C.D. at $5 \%$ & 3.94 & 3.63 & 2.22 & 3.00 & 2.93 & 2.24 \\
\hline
\end{tabular}

Note: $\quad \mathrm{FYM}-5 \mathrm{t} \mathrm{ha}^{-1} ; \mathrm{N}_{1}-100 \mathrm{~kg} \mathrm{ha}^{-1} ; \mathrm{N}_{2}-125 \mathrm{~kg} \mathrm{ha}^{-1} ; \mathrm{N}_{3^{-}} 150 \mathrm{~kg} \mathrm{ha}^{-1} ; \mathrm{P}_{1}-50 \mathrm{~kg} \mathrm{ha}^{-1} ; \mathrm{P}_{2}-75 \mathrm{~kg} \mathrm{ha}^{-1} ; \mathrm{K}_{1}-50 \mathrm{~kg} \mathrm{ha}^{-1}$; $\mathrm{K}_{2}-75 \mathrm{~kg} \mathrm{ha}^{-1} ; \mathrm{K}_{3}-100 \mathrm{~kg} \mathrm{ha}^{-1}$; NS - Non significant ; DAS - Days after sowing 
T.V. Jyothi and N.S. Hebsur / J. Appl. \& Nat. Sci. 9 (4): 2315 - 2326 (2017)

Table 3. Phosphorus uptake by Bt cotton as influenced by different levels of NPK at 60 and 90 DAS in Alfisol.

\begin{tabular}{|c|c|c|c|c|c|c|}
\hline \multirow{3}{*}{ Treatments } & \multicolumn{6}{|c|}{ Phosphorus uptake $\left(\mathrm{kg} \mathrm{ha}^{-1}\right)$} \\
\hline & \multicolumn{3}{|c|}{ 60 DAS } & \multicolumn{3}{|c|}{90 DAS } \\
\hline & $2012-13$ & 2013-14 & Pooled & 2012-13 & 2013-14 & Pooled \\
\hline $\mathrm{N}_{1}$ & 5.36 & 5.77 & 5.57 & 12.36 & 14.73 & 13.55 \\
\hline $\mathrm{N}_{2}$ & 5.46 & 6.01 & 5.74 & 15.81 & 16.86 & 16.34 \\
\hline $\mathrm{N}_{3}$ & 5.53 & 5.93 & 5.73 & 17.23 & 19.19 & 18.21 \\
\hline S.Em. \pm & 0.02 & 0.05 & 0.03 & 0.20 & 0.27 & 0.19 \\
\hline C.D. at $5 \%$ & 0.05 & 0.14 & 0.08 & 0.56 & 0.78 & 0.55 \\
\hline $\mathrm{P}_{1}$ & 5.46 & 5.93 & 5.69 & 14.91 & 16.79 & 15.85 \\
\hline $\mathrm{P}_{2}$ & 5.45 & 5.87 & 5.66 & 15.37 & 17.07 & 16.22 \\
\hline S.Em. \pm & 0.01 & 0.04 & 0.02 & 0.16 & 0.22 & 0.16 \\
\hline C.D. at $5 \%$ & NS & NS & NS & NS & NS & NS \\
\hline $\mathrm{K}_{1}$ & 5.41 & 5.82 & 5.61 & 14.42 & 16.46 & 15.44 \\
\hline $\mathrm{K}_{2}$ & 5.46 & 5.93 & 5.70 & 15.40 & 17.09 & 16.24 \\
\hline $\mathrm{K}_{3}$ & 5.49 & 5.96 & 5.72 & 15.59 & 17.23 & 16.41 \\
\hline S.Em. \pm & 0.02 & 0.05 & 0.03 & 0.20 & 0.27 & 0.19 \\
\hline C.D. at $5 \%$ & 0.05 & NS & 0.08 & 0.56 & NS & 0.55 \\
\hline $\mathrm{N}_{1} \mathrm{P}_{1}$ & 5.35 & 5.73 & 5.54 & 11.53 & 14.06 & 12.79 \\
\hline $\mathrm{N}_{1} \mathrm{P}_{2}$ & 5.37 & 5.81 & 5.59 & 13.20 & 15.41 & 14.30 \\
\hline $\mathrm{N}_{2} \mathrm{P}_{1}$ & 5.42 & 5.97 & 5.70 & 15.45 & 16.00 & 15.72 \\
\hline $\mathrm{N}_{2} \mathrm{P}_{2}$ & 5.50 & 6.05 & 5.78 & 16.18 & 17.72 & 16.95 \\
\hline $\mathrm{N}_{3} \mathrm{P}_{1}$ & 5.59 & 6.09 & 5.84 & 17.75 & 20.31 & 19.03 \\
\hline $\mathrm{N}_{3} \mathrm{P}_{2}$ & 5.47 & 5.77 & 5.62 & 16.72 & 18.07 & 17.39 \\
\hline S.Em. \pm & 0.02 & 0.07 & 0.04 & 0.28 & 0.38 & 0.27 \\
\hline C.D. at $5 \%$ & 0.07 & 0.20 & 0.11 & 0.80 & 1.10 & 0.78 \\
\hline $\mathrm{N}_{1} \mathrm{~K}_{1}$ & 5.31 & 5.53 & 5.42 & 11.27 & 13.83 & 12.55 \\
\hline $\mathrm{N}_{1} \mathrm{~K}_{2}$ & 5.36 & 5.82 & 5.59 & 12.56 & 15.24 & 13.90 \\
\hline $\mathrm{N}_{1} \mathrm{~K}_{3}$ & 5.42 & 5.96 & 5.69 & 13.27 & 15.13 & 14.20 \\
\hline $\mathrm{N}_{2} \mathrm{~K}_{1}$ & 5.36 & 5.95 & 5.65 & 15.14 & 16.17 & 15.65 \\
\hline $\mathrm{N}_{2} \mathrm{~K}_{2}$ & 5.48 & 6.02 & 5.75 & 15.68 & 16.23 & 15.96 \\
\hline $\mathrm{N}_{2} \mathrm{~K}_{3}$ & 5.55 & 6.06 & 5.81 & 16.62 & 18.18 & 17.40 \\
\hline $\mathrm{N}_{3} \mathrm{~K}_{1}$ & 5.55 & 5.97 & 5.76 & 16.86 & 19.39 & 18.13 \\
\hline $\mathrm{N}_{3} \mathrm{~K}_{2}$ & 5.54 & 5.96 & 5.75 & 17.95 & 19.79 & 18.87 \\
\hline $\mathrm{N}_{3} \mathrm{~K}_{3}$ & 5.50 & 5.85 & 5.68 & 16.90 & 18.39 & 17.64 \\
\hline S.Em. \pm & 0.03 & 0.09 & 0.05 & 0.34 & 0.47 & 0.33 \\
\hline C.D. at $5 \%$ & 0.09 & NS & 0.14 & 0.98 & 1.35 & 0.95 \\
\hline $\mathrm{P}_{1} \mathrm{~K}_{1}$ & 5.39 & 5.83 & 5.61 & 13.86 & 16.03 & 14.94 \\
\hline $\mathrm{P}_{1} \mathrm{~K}_{2}$ & 5.47 & 5.95 & 5.71 & 15.51 & 17.49 & 16.50 \\
\hline $\mathrm{P}_{1} \mathrm{~K}_{3}$ & 5.51 & 6.00 & 5.75 & 15.36 & 16.85 & 16.10 \\
\hline $\mathrm{P}_{2} \mathrm{~K}_{1}$ & 5.42 & 5.80 & 5.61 & 14.98 & 16.90 & 15.94 \\
\hline $\mathrm{P}_{2} \mathrm{~K}_{2}$ & 5.45 & 5.91 & 5.68 & 15.28 & 16.68 & 15.98 \\
\hline $\mathrm{P}_{2} \mathrm{~K}_{3}$ & 5.47 & 5.91 & 5.69 & 15.83 & 17.62 & 16.73 \\
\hline S.Em. \pm & 0.02 & 0.07 & 0.04 & 0.28 & 0.38 & 0.27 \\
\hline C.D. at $5 \%$ & NS & NS & NS & NS & NS & 0.78 \\
\hline $\mathrm{N}_{1} \mathrm{P}_{1} \mathrm{~K}_{1}$ & 5.27 & 5.50 & 5.39 & 9.85 & 12.87 & 11.36 \\
\hline $\mathrm{N}_{1} \mathrm{P}_{1} \mathrm{~K}_{2}$ & 5.35 & 5.74 & 5.54 & 11.98 & 14.54 & 13.26 \\
\hline $\mathrm{N}_{1} \mathrm{P}_{1} \mathrm{~K}_{3}$ & 5.43 & 5.95 & 5.69 & 12.75 & 14.78 & 13.76 \\
\hline $\mathrm{N}_{1} \mathrm{P}_{2} \mathrm{~K}_{1}$ & 5.35 & 5.56 & 5.45 & 12.68 & 14.79 & 13.74 \\
\hline $\mathrm{N}_{1} \mathrm{P}_{2} \mathrm{~K}_{2}$ & 5.38 & 5.90 & 5.64 & 13.14 & 15.95 & 14.54 \\
\hline $\mathrm{N}_{1} \mathrm{P}_{2} \mathrm{~K}_{3}$ & 5.40 & 5.97 & 5.68 & 13.78 & 15.49 & 14.64 \\
\hline $\mathrm{N}_{2} \mathrm{P}_{1} \mathrm{~K}_{1}$ & 5.30 & 5.92 & 5.61 & 14.93 & 15.72 & 15.33 \\
\hline $\mathrm{N}_{2} \mathrm{P}_{1} \mathrm{~K}_{2}$ & 5.44 & 5.97 & 5.71 & 15.45 & 16.06 & 15.76 \\
\hline $\mathrm{N}_{2} \mathrm{P}_{1} \mathrm{~K}_{3}$ & 5.53 & 6.03 & 5.78 & 15.97 & 16.21 & 16.09 \\
\hline $\mathrm{N}_{2} \mathrm{P}_{2} \mathrm{~K}_{1}$ & 5.42 & 5.97 & 5.70 & 15.34 & 16.62 & 15.98 \\
\hline $\mathrm{N}_{2} \mathrm{P}_{2} \mathrm{~K}_{2}$ & 5.52 & 6.08 & 5.80 & 15.92 & 16.40 & 16.16 \\
\hline $\mathrm{N}_{2} \mathrm{P}_{2} \mathrm{~K}_{3}$ & 5.57 & 6.10 & 5.83 & 17.27 & 20.15 & 18.71 \\
\hline $\mathrm{N}_{3} \mathrm{P}_{1} \mathrm{~K}_{1}$ & 5.60 & 6.09 & 5.84 & 16.79 & 19.50 & 18.15 \\
\hline $\mathrm{N}_{3} \mathrm{P}_{1} \mathrm{~K}_{2}$ & 5.63 & 6.16 & 5.89 & 19.11 & 21.88 & 20.49 \\
\hline $\mathrm{N}_{3} \mathrm{P}_{1} \mathrm{~K}_{3}$ & 5.55 & 6.03 & 5.79 & 17.36 & 19.55 & 18.45 \\
\hline $\mathrm{N}_{3} \mathrm{P}_{2} \mathrm{~K}_{1}$ & 5.50 & 5.85 & 5.68 & 16.93 & 19.28 & 18.10 \\
\hline $\mathrm{N}_{3} \mathrm{P}_{2} \mathrm{~K}_{2}$ & 5.45 & 5.77 & 5.61 & 16.79 & 17.69 & 17.24 \\
\hline $\mathrm{N}_{3} \mathrm{P}_{2} \mathrm{~K}_{3}$ & 5.45 & 5.68 & 5.56 & 16.44 & 17.24 & 16.84 \\
\hline S.Em. \pm & 0.04 & 0.12 & 0.07 & 0.48 & 0.66 & 0.47 \\
\hline C.D. at $5 \%$ & NS & NS & NS & 1.38 & 1.90 & 1.34 \\
\hline Control & 5.08 & 5.15 & 5.12 & 5.96 & 7.67 & 6.82 \\
\hline S.Em. \pm & 0.04 & 0.12 & 0.07 & 0.48 & 0.65 & 0.46 \\
\hline C.D. at $5 \%$ & 0.12 & 0.34 & 0.19 & 1.38 & 1.86 & 1.32 \\
\hline
\end{tabular}

Note: $\quad \mathrm{FYM}-5 \mathrm{t} \mathrm{ha}^{-1} ; \mathrm{N}_{1}-100 \mathrm{~kg} \mathrm{ha}^{-1} ; \mathrm{N}_{2}-125 \mathrm{~kg} \mathrm{ha}^{-1} ; \mathrm{N}_{3^{-}} 150 \mathrm{~kg} \mathrm{ha}^{-1} ; \mathrm{P}_{1}-50 \mathrm{~kg} \mathrm{ha}^{-1} ; \mathrm{P}_{2}-75 \mathrm{~kg} \mathrm{ha}^{-1} ; \mathrm{K}_{1}-50 \mathrm{~kg} \mathrm{ha}^{-1}$; $\mathrm{K}_{2}-75 \mathrm{~kg} \mathrm{ha}^{-1} ; \mathrm{K}_{3}-100 \mathrm{~kg} \mathrm{ha}^{-1}$; NS - Non significant ; DAS - Days after sowing. 
T.V. Jyothi and N.S. Hebsur / J. Appl. \& Nat. Sci. 9 (4): 2315 - 2326 (2017)

Table 4. Phosphorus uptake by Bt cotton as influenced by different levels of NPK at 120 DAS and harvest in Alfisol.

\begin{tabular}{|c|c|c|c|c|c|c|}
\hline \multirow{3}{*}{ Treatments } & \multicolumn{6}{|c|}{ Phosphorus uptake $\left(\mathrm{kg} \mathrm{ha}^{-1}\right)$} \\
\hline & \multicolumn{3}{|c|}{120 DAS } & \multicolumn{3}{|c|}{ At harvest } \\
\hline & 2012-13 & 2013-14 & Pooled & 2012-13 & 2013-14 & Pooled \\
\hline $\mathrm{N}_{1}$ & 19.82 & 20.98 & 20.40 & 21.19 & 22.79 & 21.99 \\
\hline $\mathrm{N}_{2}$ & 23.26 & 24.12 & 23.69 & 24.57 & 26.59 & 25.58 \\
\hline $\mathrm{N}_{3}$ & 25.14 & 27.36 & 26.25 & 27.87 & 30.26 & 29.07 \\
\hline S.Em. \pm & 0.52 & 0.45 & 0.38 & 0.32 & 0.26 & 0.20 \\
\hline C.D. at $5 \%$ & 1.50 & 1.29 & 1.08 & 0.92 & 0.74 & 0.59 \\
\hline $\mathrm{P}_{1}$ & 22.43 & 23.91 & 23.17 & 24.42 & 26.23 & 25.32 \\
\hline $\mathrm{P}_{2}$ & 23.06 & 24.40 & 23.73 & 24.67 & 26.86 & 25.76 \\
\hline S.Em. \pm & 0.43 & 0.37 & 0.31 & 0.26 & 0.21 & 0.17 \\
\hline C.D. at $5 \%$ & NS & NS & NS & NS & 0.60 & NS \\
\hline $\mathrm{K}_{1}$ & 21.64 & 23.31 & 22.47 & 23.99 & 25.68 & 24.84 \\
\hline $\mathrm{K}_{2}$ & 22.44 & 24.97 & 23.71 & 24.51 & 26.47 & 25.49 \\
\hline $\mathrm{K}_{3}$ & 24.14 & 24.17 & 24.16 & 25.12 & 27.49 & 26.30 \\
\hline S.Em. \pm & 0.52 & 0.45 & 0.38 & 0.32 & 0.26 & 0.20 \\
\hline C.D. at $5 \%$ & 1.50 & 1.29 & 1.08 & NS & 0.74 & 0.59 \\
\hline $\mathrm{N}_{1} \mathrm{P}_{1}$ & 19.60 & 20.73 & 20.16 & 20.81 & 22.23 & 21.52 \\
\hline $\mathrm{N}_{1} \mathrm{P}_{2}$ & 20.04 & 21.24 & 20.64 & 21.56 & 23.34 & 22.45 \\
\hline $\mathrm{N}_{2} \mathrm{P}_{1}$ & 21.89 & 23.52 & 22.70 & 23.81 & 25.43 & 24.62 \\
\hline $\mathrm{N}_{2} \mathrm{P}_{2}$ & 24.64 & 24.71 & 24.68 & 25.33 & 27.75 & 26.54 \\
\hline $\mathrm{N}_{3} \mathrm{P}_{1}$ & 25.79 & 27.48 & 26.63 & 28.63 & 31.03 & 29.83 \\
\hline $\mathrm{N}_{3} \mathrm{P}_{2}$ & 24.50 & 27.24 & 25.87 & 27.12 & 29.49 & 28.30 \\
\hline S.Em. \pm & 0.74 & 0.64 & 0.53 & 0.45 & 0.36 & 0.29 \\
\hline C.D. at $5 \%$ & 2.12 & NS & 1.52 & 1.30 & 1.04 & 0.83 \\
\hline $\mathrm{N}_{1} \mathrm{~K}_{1}$ & 18.64 & 19.61 & 19.12 & 20.11 & 21.66 & 20.88 \\
\hline $\mathrm{N}_{1} \mathrm{~K}_{2}$ & 19.78 & 21.12 & 20.45 & 21.29 & 22.50 & 21.90 \\
\hline $\mathrm{N}_{1} \mathrm{~K}_{3}$ & 21.04 & 22.22 & 21.63 & 22.17 & 24.19 & 23.18 \\
\hline $\mathrm{N}_{2} \mathrm{~K}_{1}$ & 21.89 & 23.19 & 22.54 & 23.65 & 25.47 & 24.56 \\
\hline $\mathrm{N}_{2} \mathrm{~K}_{2}$ & 22.58 & 24.17 & 23.37 & 23.72 & 25.97 & 24.84 \\
\hline $\mathrm{N}_{2} \mathrm{~K}_{3}$ & 25.32 & 24.98 & 25.15 & 26.34 & 28.34 & 27.34 \\
\hline $\mathrm{N}_{3} \mathrm{~K}_{1}$ & 24.39 & 27.13 & 25.76 & 28.21 & 29.91 & 29.06 \\
\hline $\mathrm{N}_{3} \mathrm{~K}_{2}$ & 24.97 & 29.63 & 27.30 & 28.54 & 30.94 & 29.74 \\
\hline $\mathrm{N}_{3} \mathrm{~K}_{3}$ & 26.07 & 25.33 & 25.70 & 26.86 & 29.93 & 28.40 \\
\hline S.Em. \pm & 0.90 & 0.78 & 0.65 & 0.56 & 0.44 & 0.35 \\
\hline C.D. at $5 \%$ & NS & 2.24 & NS & 1.60 & 1.28 & 1.02 \\
\hline $\mathrm{P}_{1} \mathrm{~K}_{1}$ & 20.45 & 23.00 & 21.73 & 23.48 & 25.03 & 24.26 \\
\hline $\mathrm{P}_{1} \mathrm{~K}_{2}$ & 23.32 & 24.96 & 24.14 & 24.51 & 26.61 & 25.56 \\
\hline $\mathrm{P}_{1} \mathrm{~K}_{3}$ & 23.51 & 23.76 & 23.63 & 25.26 & 27.05 & 26.16 \\
\hline $\mathrm{P}_{2} \mathrm{~K}_{1}$ & 22.83 & 23.62 & 23.22 & 24.50 & 26.33 & 25.42 \\
\hline $\mathrm{P}_{2} \mathrm{~K}_{2}$ & 21.56 & 24.99 & 23.28 & 24.52 & 26.33 & 25.43 \\
\hline $\mathrm{P}_{2} \mathrm{~K}_{3}$ & 24.78 & 24.59 & 24.69 & 24.98 & 27.92 & 26.45 \\
\hline S.Em.土 & 0.74 & 0.64 & 0.53 & 0.79 & 0.36 & 0.29 \\
\hline C.D. at $5 \%$ & 2.12 & NS & NS & NS & NS & NS \\
\hline $\mathrm{N}_{1} \mathrm{P}_{1} \mathrm{~K}_{1}$ & 18.29 & 19.56 & 18.92 & 19.49 & 20.78 & 20.14 \\
\hline $\mathrm{N}_{1} \mathrm{P}_{1} \mathrm{~K}_{2}$ & 19.83 & 20.60 & 20.21 & 20.96 & 22.38 & 21.67 \\
\hline $\mathrm{N}_{1} \mathrm{P}_{1} \mathrm{~K}_{3}$ & 20.69 & 22.02 & 21.36 & 21.99 & 23.52 & 22.76 \\
\hline $\mathrm{N}_{1} \mathrm{P}_{2} \mathrm{~K}_{1}$ & 18.99 & 19.66 & 19.32 & 20.73 & 22.54 & 21.63 \\
\hline $\mathrm{N}_{1} \mathrm{P}_{2} \mathrm{~K}_{2}$ & 19.73 & 21.64 & 20.69 & 21.61 & 22.63 & 22.12 \\
\hline $\mathrm{N}_{1} \mathrm{P}_{2} \mathrm{~K}_{3}$ & 21.38 & 22.41 & 21.90 & 22.34 & 24.86 & 23.60 \\
\hline $\mathrm{N}_{2} \mathrm{P}_{1} \mathrm{~K}_{1}$ & 20.63 & 21.62 & 21.12 & 22.79 & 24.56 & 23.68 \\
\hline $\mathrm{N}_{2} \mathrm{P}_{1} \mathrm{~K}_{2}$ & 21.68 & 23.44 & 22.56 & 22.65 & 24.84 & 23.74 \\
\hline $\mathrm{N}_{2} \mathrm{P}_{1} \mathrm{~K}_{3}$ & 23.35 & 25.51 & 24.43 & 25.98 & 26.91 & 26.45 \\
\hline $\mathrm{N}_{2} \mathrm{P}_{2} \mathrm{~K}_{1}$ & 23.16 & 24.77 & 23.97 & 24.52 & 26.38 & 25.45 \\
\hline $\mathrm{N}_{2} \mathrm{P}_{2} \mathrm{~K}_{2}$ & 23.48 & 24.91 & 24.19 & 24.78 & 27.10 & 25.94 \\
\hline $\mathrm{N}_{2} \mathrm{P}_{2} \mathrm{~K}_{3}$ & 27.28 & 24.45 & 25.87 & 26.69 & 29.77 & 28.23 \\
\hline $\mathrm{N}_{3} \mathrm{P}_{1} \mathrm{~K}_{1}$ & 22.43 & 27.83 & 25.13 & 28.17 & 29.75 & 28.96 \\
\hline $\mathrm{N}_{3} \mathrm{P}_{1} \mathrm{~K}_{2}$ & 28.46 & 30.84 & 29.65 & 29.91 & 32.61 & 31.26 \\
\hline $\mathrm{N}_{3} \mathrm{P}_{1} \mathrm{~K}_{3}$ & 26.47 & 23.75 & 25.11 & 27.80 & 30.74 & 29.27 \\
\hline $\mathrm{N}_{3} \mathrm{P}_{2} \mathrm{~K}_{1}$ & 26.34 & 26.42 & 26.38 & 28.26 & 30.06 & 29.16 \\
\hline $\mathrm{N}_{3} \mathrm{P}_{2} \mathrm{~K}_{2}$ & 21.48 & 28.41 & 24.95 & 27.17 & 29.27 & 28.22 \\
\hline $\mathrm{N}_{3} \mathrm{P}_{2} \mathrm{~K}_{3}$ & 25.67 & 26.90 & 26.29 & 25.92 & 29.13 & 27.53 \\
\hline S.Em. \pm & 1.28 & 1.10 & 0.92 & 0.79 & 0.63 & 0.50 \\
\hline C.D. at $5 \%$ & 3.67 & 3.17 & 2.64 & 2.26 & 1.80 & 1.44 \\
\hline Control & 10.74 & 11.43 & 11.09 & 16.88 & 17.30 & 17.09 \\
\hline S.Em.土 & 1.25 & 1.08 & 0.89 & 0.76 & 0.61 & 0.49 \\
\hline C.D. at $5 \%$ & 3.58 & 3.09 & 2.56 & 2.19 & 1.76 & 1.40 \\
\hline
\end{tabular}

Note: $\quad \mathrm{FYM}-5 \mathrm{t} \mathrm{ha}^{-1} ; \mathrm{N}_{1}-100 \mathrm{~kg} \mathrm{ha}^{-1} ; \mathrm{N}_{2}-125 \mathrm{~kg} \mathrm{ha}^{-1} ; \mathrm{N}_{3}-150 \mathrm{~kg} \mathrm{ha}^{-1} ; \mathrm{P}_{1}-50 \mathrm{~kg} \mathrm{ha}^{-1} ; \mathrm{P}_{2}-75 \mathrm{~kg} \mathrm{ha}^{-1} ; \mathrm{K}_{1}-50 \mathrm{~kg} \mathrm{ha}^{-1}$; $\mathrm{K}_{2}-75 \mathrm{~kg} \mathrm{ha}^{-1} ; \mathrm{K}_{3}-100 \mathrm{~kg} \mathrm{ha}^{-1} ; \mathrm{NS}-$ Non significant ; DAS - Days after sowing. 
T.V. Jyothi and N.S. Hebsur / J. Appl. \& Nat. Sci. 9 (4): 2315 - 2326 (2017)

Table 5. Potassium uptake by Bt cotton as influenced by different levels of NPK at 60 and 90 DAS in Alfisol.

\begin{tabular}{|c|c|c|c|c|c|c|}
\hline \multirow{3}{*}{ Treatments } & \multicolumn{6}{|c|}{ Potassium uptake $\left(\mathrm{kg} \mathrm{ha}^{-1}\right)$} \\
\hline & \multicolumn{3}{|c|}{60 DAS } & \multicolumn{3}{|c|}{90 DAS } \\
\hline & 2012-13 & 2013-14 & Pooled & 2012-13 & 2013-14 & Pooled \\
\hline $\mathrm{N}_{1}$ & 26.44 & 27.86 & 27.15 & 70.73 & 72.14 & 71.44 \\
\hline $\mathrm{N}_{2}$ & 28.52 & 30.03 & 29.27 & 73.63 & 77.05 & 75.34 \\
\hline $\mathrm{N}_{3}$ & 30.18 & 31.74 & 30.96 & 77.39 & 79.43 & 78.41 \\
\hline S.Em. \pm & 0.29 & 0.32 & 0.22 & 0.56 & 0.57 & 0.40 \\
\hline C.D. at $5 \%$ & 0.84 & 0.91 & 0.62 & 1.62 & 1.65 & 1.15 \\
\hline $\mathrm{P}_{1}$ & 28.38 & 30.11 & 29.25 & 73.36 & 76.46 & 74.91 \\
\hline $\mathrm{P}_{2}$ & 28.38 & 29.64 & 29.01 & 74.47 & 75.96 & 75.22 \\
\hline S.Em. \pm & 0.24 & 0.26 & 0.18 & 0.46 & 0.47 & 0.33 \\
\hline C.D. at $5 \%$ & NS & NS & NS & NS & NS & NS \\
\hline $\mathrm{K}_{1}$ & 27.40 & 28.80 & 28.10 & 72.23 & 75.21 & 73.72 \\
\hline $\mathrm{K}_{2}$ & 28.40 & 29.84 & 29.12 & 74.22 & 76.59 & 75.26 \\
\hline $\mathrm{K}_{3}$ & 29.33 & 30.99 & 30.16 & 75.30 & 77.12 & 76.21 \\
\hline S.Em. \pm & 0.29 & 0.32 & 0.22 & 0.56 & 0.57 & 0.40 \\
\hline C.D. at $5 \%$ & 0.84 & 0.91 & 0.62 & 1.62 & NS & 1.15 \\
\hline $\mathrm{N}_{1} \mathrm{P}_{1}$ & 26.21 & 27.62 & 26.91 & 70.56 & 71.96 & 71.26 \\
\hline $\mathrm{N}_{1} \mathrm{P}_{2}$ & 26.67 & 28.10 & 27.38 & 70.91 & 72.32 & 71.61 \\
\hline $\mathrm{N}_{2} \mathrm{P}_{1}$ & 28.24 & 29.99 & 29.12 & 71.79 & 76.29 & 74.04 \\
\hline $\mathrm{N}_{2} \mathrm{P}_{2}$ & 28.79 & 30.07 & 29.43 & 75.47 & 77.81 & 76.64 \\
\hline $\mathrm{N}_{3} \mathrm{P}_{1}$ & 30.68 & 32.73 & 31.70 & 77.74 & 81.13 & 79.43 \\
\hline $\mathrm{N}_{3} \mathrm{P}_{2}$ & 29.68 & 30.76 & 30.22 & 77.05 & 77.74 & 77.39 \\
\hline S.Em. \pm & 0.41 & 0.45 & 0.31 & 0.80 & 0.81 & 0.57 \\
\hline C.D. at $5 \%$ & NS & 1.28 & 0.88 & 2.29 & 2.33 & 1.62 \\
\hline $\mathrm{N}_{1} \mathrm{~K}_{1}$ & 25.20 & 26.43 & 25.81 & 68.09 & 69.85 & 68.97 \\
\hline $\mathrm{N}_{1} \mathrm{~K}_{2}$ & 26.12 & 27.57 & 26.84 & 70.79 & 72.53 & 71.66 \\
\hline $\mathrm{N}_{1} \mathrm{~K}_{3}$ & 28.00 & 29.58 & 28.79 & 73.32 & 74.04 & 73.68 \\
\hline $\mathrm{N}_{2} \mathrm{~K}_{1}$ & 27.14 & 28.38 & 27.76 & 71.91 & 77.06 & 74.48 \\
\hline $\mathrm{N}_{2} \mathrm{~K}_{2}$ & 28.32 & 29.51 & 28.91 & 73.72 & 75.67 & 74.70 \\
\hline $\mathrm{N}_{2} \mathrm{~K}_{3}$ & 30.08 & 32.21 & 31.15 & 75.26 & 78.43 & 76.85 \\
\hline $\mathrm{N}_{3} \mathrm{~K}_{1}$ & 29.87 & 31.60 & 30.74 & 76.69 & 78.74 & 77.72 \\
\hline $\mathrm{N}_{3} \mathrm{~K}_{2}$ & 30.76 & 32.45 & 31.61 & 78.17 & 80.66 & 79.41 \\
\hline $\mathrm{N}_{3} \mathrm{~K}_{3}$ & 29.90 & 31.18 & 30.54 & 77.32 & 78.90 & 78.11 \\
\hline S.Em. \pm & 0.50 & 0.55 & 0.38 & 0.98 & 0.99 & 0.69 \\
\hline C.D. at $5 \%$ & 1.45 & 1.57 & 1.08 & NS & NS & 1.99 \\
\hline $\mathrm{P}_{1} \mathrm{~K}_{1}$ & 27.05 & 28.38 & 27.72 & 71.23 & 75.83 & 73.53 \\
\hline $\mathrm{P}_{1} \mathrm{~K}_{2}$ & 28.71 & 30.75 & 29.73 & 73.85 & 76.54 & 75.20 \\
\hline $\mathrm{P}_{1} \mathrm{~K}_{3}$ & 29.37 & 31.21 & 30.29 & 75.01 & 77.00 & 76.01 \\
\hline $\mathrm{P}_{2} \mathrm{~K}_{1}$ & 27.76 & 29.22 & 28.49 & 73.23 & 74.59 & 73.91 \\
\hline $\mathrm{P}_{2} \mathrm{~K}_{2}$ & 28.09 & 28.94 & 28.52 & 74.60 & 76.03 & 75.31 \\
\hline $\mathrm{P}_{2} \mathrm{~K}_{3}$ & 29.28 & 30.77 & 30.02 & 75.59 & 77.24 & 76.42 \\
\hline S.Em. \pm & 0.41 & 0.45 & 0.31 & 0.80 & 0.81 & 0.57 \\
\hline C.D. at $5 \%$ & NS & 1.28 & 0.88 & NS & NS & NS \\
\hline $\mathrm{N}_{1} \mathrm{P}_{1} \mathrm{~K}_{1}$ & 24.71 & 25.50 & 25.10 & 66.30 & 69.57 & 67.94 \\
\hline $\mathrm{N}_{1} \mathrm{P}_{1} \mathrm{~K}_{2}$ & 25.98 & 28.02 & 27.00 & 70.68 & 72.16 & 71.42 \\
\hline $\mathrm{N}_{1} \mathrm{P}_{1} \mathrm{~K}_{3}$ & 27.94 & 29.35 & 28.64 & 74.70 & 74.14 & 74.42 \\
\hline $\mathrm{N}_{1} \mathrm{P}_{2} \mathrm{~K}_{1}$ & 25.69 & 27.35 & 26.52 & 69.88 & 70.12 & 70.00 \\
\hline $\mathrm{N}_{1} \mathrm{P}_{2} \mathrm{~K}_{2}$ & 26.25 & 27.11 & 26.68 & 70.89 & 72.90 & 71.89 \\
\hline $\mathrm{N}_{1} \mathrm{P}_{2} \mathrm{~K}_{3}$ & 28.06 & 29.82 & 28.94 & 71.94 & 73.94 & 72.94 \\
\hline $\mathrm{N}_{2} \mathrm{P}_{1} \mathrm{~K}_{1}$ & 26.92 & 28.14 & 27.53 & 70.49 & 78.66 & 74.57 \\
\hline $\mathrm{N}_{2} \mathrm{P}_{1} \mathrm{~K}_{2}$ & 28.35 & 29.69 & 29.02 & 71.65 & 73.82 & 72.73 \\
\hline $\mathrm{N}_{2} \mathrm{P}_{1} \mathrm{~K}_{3}$ & 29.45 & 32.15 & 30.80 & 73.25 & 76.41 & 74.83 \\
\hline $\mathrm{N}_{2} \mathrm{P}_{2} \mathrm{~K}_{1}$ & 27.37 & 28.62 & 27.99 & 73.33 & 75.45 & 74.39 \\
\hline $\mathrm{N}_{2} \mathrm{P}_{2} \mathrm{~K}_{2}$ & 28.29 & 29.32 & 28.81 & 75.79 & 77.53 & 76.66 \\
\hline $\mathrm{N}_{2} \mathrm{P}_{2} \mathrm{~K}_{3}$ & 30.72 & 32.27 & 31.49 & 77.28 & 80.46 & 78.87 \\
\hline $\mathrm{N}_{3} \mathrm{P}_{1} \mathrm{~K}_{1}$ & 29.52 & 31.51 & 30.51 & 76.90 & 79.27 & 78.09 \\
\hline $\mathrm{N}_{3} \mathrm{P}_{1} \mathrm{~K}_{2}$ & 31.79 & 34.53 & 33.16 & 79.24 & 83.64 & 81.44 \\
\hline $\mathrm{N}_{3} \mathrm{P}_{1} \mathrm{~K}_{3}$ & 30.73 & 32.15 & 31.44 & 77.08 & 80.47 & 78.77 \\
\hline $\mathrm{N}_{3} \mathrm{P}_{2} \mathrm{~K}_{1}$ & 30.22 & 31.69 & 30.96 & 76.48 & 78.21 & 77.34 \\
\hline $\mathrm{N}_{3} \mathrm{P}_{2} \mathrm{~K}_{2}$ & 29.74 & 30.38 & 30.06 & 77.10 & 77.67 & 77.39 \\
\hline $\mathrm{N}_{3} \mathrm{P}_{2} \mathrm{~K}_{3}$ & 29.07 & 30.21 & 29.64 & 77.56 & 77.33 & 77.45 \\
\hline S.Em. \pm & 0.71 & 0.77 & 0.53 & 1.38 & 1.41 & 0.98 \\
\hline C.D. at $5 \%$ & NS & NS & NS & NS & 4.04 & 2.81 \\
\hline Control & 19.52 & 20.16 & 19.84 & 48.08 & 50.14 & 49.11 \\
\hline S.Em. \pm & 0.70 & 0.77 & 0.52 & 1.47 & 1.38 & 0.99 \\
\hline C.D. at $5 \%$ & 2.00 & 2.21 & 1.49 & 4.20 & 3.95 & 2.83 \\
\hline
\end{tabular}

Note: $\quad \mathrm{FYM}-5 \mathrm{t} \mathrm{ha}^{-1} ; \mathrm{N}_{1}-100 \mathrm{~kg} \mathrm{ha}^{-1} ; \mathrm{N}_{2}-125 \mathrm{~kg} \mathrm{ha}^{-1} ; \mathrm{N}_{3}-150 \mathrm{~kg} \mathrm{ha}^{-1} ; \mathrm{P}_{1}-50 \mathrm{~kg} \mathrm{ha}^{-1} ; \mathrm{P}_{2}-75 \mathrm{~kg} \mathrm{ha}^{-1} ; \mathrm{K}_{1}-50 \mathrm{~kg} \mathrm{ha}^{-1}$; $\mathrm{K}_{2}-75 \mathrm{~kg} \mathrm{ha}^{-1} ; \mathrm{K}_{3}-100 \mathrm{~kg} \mathrm{ha}^{-1} ; \mathrm{NS}-$ Non significant ; DAS - Days after sowing. 
T.V. Jyothi and N.S. Hebsur / J. Appl. \& Nat. Sci. 9 (4): 2315 - 2326 (2017)

Table 6. Potassium uptake by Bt cotton as influenced by different levels of NPK at 120 DAS and harvest in Alfisol.

\begin{tabular}{|c|c|c|c|c|c|c|}
\hline \multirow{3}{*}{ Treatments } & \multicolumn{6}{|c|}{ Potassium uptake $\left(\mathrm{kg} \mathrm{ha}^{-1}\right)$} \\
\hline & \multicolumn{3}{|c|}{120 DAS } & \multicolumn{3}{|c|}{ At harvest } \\
\hline & $2012-13$ & 2013-14 & Pooled & $2012-13$ & 2013-14 & Pooled \\
\hline$\overline{\mathrm{N}_{1}}$ & 114.26 & 117.01 & 115.64 & 115.95 & 118.13 & 117.04 \\
\hline $\mathrm{N}_{2}$ & 118.69 & 121.43 & 120.06 & 120.24 & 122.33 & 121.28 \\
\hline $\mathrm{N}_{3}$ & 122.68 & 125.14 & 123.91 & 123.81 & 126.92 & 125.37 \\
\hline S.Em. \pm & 0.60 & 1.29 & 0.69 & 0.38 & 0.41 & 0.28 \\
\hline C.D. at $5 \%$ & 1.71 & 3.69 & 1.98 & 1.09 & 1.16 & 0.82 \\
\hline $\mathrm{P}_{1}$ & 117.87 & 120.29 & 119.08 & 119.21 & 121.53 & 120.37 \\
\hline $\mathrm{P}_{2}$ & 119.21 & 122.10 & 120.66 & 120.79 & 123.39 & 122.09 \\
\hline S.Em. \pm & 0.49 & 1.05 & 0.56 & 0.31 & 0.33 & 0.23 \\
\hline C.D. at $5 \%$ & NS & NS & NS & 0.89 & 0.95 & 0.67 \\
\hline $\mathrm{K}_{1}$ & 117.10 & 120.00 & 118.55 & 118.70 & 120.65 & 119.68 \\
\hline $\mathrm{K}_{2}$ & 118.83 & 121.30 & 120.06 & 120.51 & 122.64 & 121.58 \\
\hline $\mathrm{K}_{3}$ & 119.70 & 122.29 & 120.99 & 120.78 & 124.08 & 122.43 \\
\hline S.Em. \pm & 0.60 & 1.29 & 0.69 & 0.38 & 0.41 & 0.28 \\
\hline C.D. at $5 \%$ & 1.71 & 3.69 & 1.98 & 1.09 & 1.16 & 0.82 \\
\hline $\mathrm{N}_{1} \mathrm{P}_{1}$ & 113.14 & 115.47 & 114.30 & 115.37 & 117.69 & 116.53 \\
\hline $\mathrm{N}_{1} \mathrm{P}_{2}$ & 115.38 & 118.56 & 116.97 & 116.53 & 118.56 & 117.55 \\
\hline $\mathrm{N}_{2} \mathrm{P}_{1}$ & 116.06 & 118.85 & 117.45 & 117.65 & 119.66 & 118.65 \\
\hline $\mathrm{N}_{2} \mathrm{P}_{2}$ & 121.32 & 124.01 & 122.66 & 122.82 & 124.99 & 123.91 \\
\hline $\mathrm{N}_{3} \mathrm{P}_{1}$ & 124.42 & 126.54 & 125.48 & 124.62 & 127.24 & 125.93 \\
\hline $\mathrm{N}_{3} \mathrm{P}_{2}$ & 120.94 & 123.74 & 122.34 & 123.01 & 126.60 & 124.81 \\
\hline S.Em. \pm & 0.84 & 1.82 & 0.97 & 0.54 & 0.57 & 0.40 \\
\hline C.D. at $5 \%$ & 2.42 & 5.22 & 2.80 & 1.54 & 1.65 & 1.15 \\
\hline $\mathrm{N}_{1} \mathrm{~K}_{1}$ & 112.50 & 115.86 & 114.18 & 115.16 & 116.85 & 116.01 \\
\hline $\mathrm{N}_{1} \mathrm{~K}_{2}$ & 114.66 & 116.82 & 115.74 & 116.10 & 117.86 & 116.98 \\
\hline $\mathrm{N}_{1} \mathrm{~K}_{3}$ & 115.62 & 118.35 & 116.98 & 116.59 & 119.67 & 118.13 \\
\hline $\mathrm{N}_{2} \mathrm{~K}_{1}$ & 116.37 & 119.41 & 117.89 & 118.13 & 120.44 & 119.29 \\
\hline $\mathrm{N}_{2} \mathrm{~K}_{2}$ & 118.31 & 121.06 & 119.69 & 120.12 & 121.71 & 120.92 \\
\hline $\mathrm{N}_{2} \mathrm{~K}_{3}$ & 121.38 & 123.82 & 122.60 & 122.46 & 124.82 & 123.64 \\
\hline $\mathrm{N}_{3} \mathrm{~K}_{1}$ & 122.42 & 124.73 & 123.57 & 122.82 & 124.67 & 123.74 \\
\hline $\mathrm{N}_{3} \mathrm{~K}_{2}$ & 123.51 & 126.01 & 124.76 & 125.32 & 128.35 & 126.84 \\
\hline $\mathrm{N}_{3} \mathrm{~K}_{3}$ & 122.11 & 124.69 & 123.40 & 123.29 & 127.74 & 125.52 \\
\hline S.Em. \pm & 1.03 & 2.23 & 1.19 & 0.66 & 0.70 & 0.49 \\
\hline C.D. at $5 \%$ & NS & NS & 3.42 & 1.89 & NS & 1.41 \\
\hline $\mathrm{P}_{1} \mathrm{~K}_{1}$ & 115.94 & 118.76 & 117.35 & 117.11 & 119.00 & 118.05 \\
\hline $\mathrm{P}_{1} \mathrm{~K}_{2}$ & 118.80 & 120.91 & 119.85 & 120.29 & 122.31 & 121.30 \\
\hline $\mathrm{P}_{1} \mathrm{~K}_{3}$ & 118.87 & 121.19 & 120.03 & 120.24 & 123.28 & 121.76 \\
\hline $\mathrm{P}_{2} \mathrm{~K}_{1}$ & 118.26 & 121.24 & 119.75 & 120.30 & 122.30 & 121.30 \\
\hline $\mathrm{P}_{2} \mathrm{~K}_{2}$ & 118.86 & 121.68 & 120.27 & 120.74 & 122.98 & 121.86 \\
\hline $\mathrm{P}_{2} \mathrm{~K}_{3}$ & 120.53 & 123.38 & 121.96 & 121.32 & 124.88 & 123.10 \\
\hline S.Em. \pm & 0.84 & 1.82 & 0.97 & 0.54 & 0.57 & 0.40 \\
\hline C.D. at $5 \%$ & NS & NS & NS & 1.54 & NS & 1.15 \\
\hline $\mathrm{N}_{1} \mathrm{P}_{1} \mathrm{~K}_{1}$ & 111.61 & 114.72 & 113.16 & 114.72 & 116.60 & 115.66 \\
\hline $\mathrm{N}_{1} \mathrm{P}_{1} \mathrm{~K}_{2}$ & 113.63 & 114.97 & 114.30 & 115.33 & 117.40 & 116.36 \\
\hline $\mathrm{N}_{1} \mathrm{P}_{1} \mathrm{~K}_{3}$ & 114.17 & 116.72 & 115.44 & 116.06 & 119.08 & 117.57 \\
\hline $\mathrm{N}_{1} \mathrm{P}_{2} \mathrm{~K}_{1}$ & 113.39 & 117.01 & 115.20 & 115.60 & 117.11 & 116.35 \\
\hline $\mathrm{N}_{1} \mathrm{P}_{2} \mathrm{~K}_{2}$ & 115.69 & 118.67 & 117.18 & 116.87 & 118.32 & 117.59 \\
\hline $\mathrm{N}_{1} \mathrm{P}_{2} \mathrm{~K}_{3}$ & 117.06 & 119.99 & 118.53 & 117.12 & 120.27 & 118.69 \\
\hline $\mathrm{N}_{2} \mathrm{P}_{1} \mathrm{~K}_{1}$ & 113.34 & 116.48 & 114.91 & 115.53 & 118.22 & 116.87 \\
\hline $\mathrm{N}_{2} \mathrm{P}_{1} \mathrm{~K}_{2}$ & 116.31 & 119.32 & 117.82 & 117.93 & 119.25 & 118.59 \\
\hline $\mathrm{N}_{2} \mathrm{P}_{1} \mathrm{~K}_{3}$ & 118.52 & 120.75 & 119.64 & 119.48 & 121.52 & 120.50 \\
\hline $\mathrm{N}_{2} \mathrm{P}_{2} \mathrm{~K}_{1}$ & 119.41 & 122.34 & 120.88 & 120.73 & 122.67 & 121.70 \\
\hline $\mathrm{N}_{2} \mathrm{P}_{2} \mathrm{~K}_{2}$ & 120.32 & 122.79 & 121.55 & 122.31 & 124.18 & 123.25 \\
\hline $\mathrm{N}_{2} \mathrm{P}_{2} \mathrm{~K}_{3}$ & 124.24 & 126.88 & 125.56 & 125.43 & 128.13 & 126.78 \\
\hline $\mathrm{N}_{3} \mathrm{P}_{1} \mathrm{~K}_{1}$ & 122.88 & 125.07 & 123.98 & 121.07 & 122.20 & 121.63 \\
\hline $\mathrm{N}_{3} \mathrm{P}_{1} \mathrm{~K}_{2}$ & 126.46 & 128.44 & 127.45 & 127.60 & 130.27 & 128.94 \\
\hline $\mathrm{N}_{3} \mathrm{P}_{1} \mathrm{~K}_{3}$ & 123.92 & 126.11 & 125.02 & 125.18 & 129.24 & 127.21 \\
\hline $\mathrm{N}_{3} \mathrm{P}_{2} \mathrm{~K}_{1}$ & 121.97 & 124.38 & 123.17 & 124.57 & 127.14 & 125.86 \\
\hline $\mathrm{N}_{3} \mathrm{P}_{2} \mathrm{~K}_{2}$ & 120.56 & 123.58 & 122.07 & 123.05 & 126.44 & 124.74 \\
\hline $\mathrm{N}_{3} \mathrm{P}_{2} \mathrm{~K}_{3}$ & 120.29 & 123.27 & 121.78 & 121.41 & 126.24 & 123.82 \\
\hline S.Em. \pm & 1.46 & 3.15 & 1.69 & 0.93 & 0.99 & 0.69 \\
\hline C.D. at $5 \%$ & 4.19 & NS & 4.84 & 2.67 & 2.85 & 2.00 \\
\hline Control & 94.81 & 98.42 & 96.61 & 109.18 & 111.41 & 110.29 \\
\hline S.Em. \pm & 1.56 & 3.07 & 1.67 & 0.92 & 1.02 & 0.72 \\
\hline C.D. at $5 \%$ & 4.49 & 8.80 & 4.80 & 2.64 & 2.94 & 2.07 \\
\hline
\end{tabular}


T.V. Jyothi and N.S. Hebsur / J. Appl. \& Nat. Sci. 9 (4): 2315 - 2326 (2017)

Table 7. Physico-chemical parameters as influenced by different levels of NPK at harvest of Bt cotton in Alfisol.

\begin{tabular}{|c|c|c|c|c|c|c|c|c|c|}
\hline \multirow{2}{*}{ Treatments } & \multicolumn{3}{|c|}{ pH (1:2.5) } & \multicolumn{3}{|c|}{$\operatorname{EC}\left(\mathrm{dSm}^{-1}\right)(1: 2.5)$} & \multicolumn{3}{|c|}{ Organic carbon $\left(\mathrm{g} \mathrm{kg}^{-1}\right)$} \\
\hline & 2012-13 & 2013-14 & Pooled & $2012-13$ & 2013-14 & Pooled & 2012-13 & 2013-14 & Pooled \\
\hline $\mathrm{N}_{1}$ & 6.48 & 5.76 & 6.12 & 0.14 & 0.13 & 0.13 & 6.12 & 5.86 & 5.99 \\
\hline $\mathrm{N}_{2}$ & 6.44 & 5.76 & 6.10 & 0.13 & 0.14 & 0.13 & 6.05 & 5.82 & 5.94 \\
\hline $\mathrm{N}_{3}$ & 6.46 & 5.78 & 6.12 & 0.13 & 0.13 & 0.13 & 6.05 & 5.81 & 5.93 \\
\hline S.Em. \pm & 0.02 & 0.02 & 0.02 & 0.004 & 0.004 & 0.003 & 0.006 & 0.008 & 0.005 \\
\hline C.D. at $5 \%$ & NS & NS & NS & NS & NS & NS & 0.018 & 0.023 & 0.015 \\
\hline $\mathrm{P}_{1}$ & 6.45 & 5.80 & 6.13 & 0.13 & 0.13 & 0.13 & 6.07 & 5.83 & 5.95 \\
\hline $\mathrm{P}_{2}$ & 6.47 & 5.73 & 6.10 & 0.14 & 0.13 & 0.13 & 6.07 & 5.83 & 5.95 \\
\hline S.Em. \pm & 0.01 & 0.02 & 0.01 & 0.004 & 0.003 & 0.002 & 0.005 & 0.007 & 0.004 \\
\hline C.D. at $5 \%$ & NS & 0.06 & NS & NS & NS & NS & NS & NS & NS \\
\hline $\mathrm{K}_{1}$ & 6.45 & 5.81 & 6.13 & 0.13 & 0.13 & 0.13 & 6.08 & 5.85 & 5.97 \\
\hline $\mathrm{K}_{2}$ & 6.44 & 5.78 & 6.11 & 0.13 & 0.14 & 0.13 & 6.07 & 5.82 & 5.95 \\
\hline $\mathrm{K}_{3}$ & 6.49 & 5.72 & 6.10 & 0.13 & 0.13 & 0.13 & 6.06 & 5.81 & 5.94 \\
\hline S.Em. \pm & 0.02 & 0.02 & 0.02 & 0.004 & 0.004 & 0.003 & 0.006 & 0.008 & 0.005 \\
\hline C.D. at $5 \%$ & NS & NS & NS & NS & NS & NS & 0.018 & 0.023 & 0.015 \\
\hline $\mathrm{N}_{1} \mathrm{P}_{1}$ & 6.49 & 5.83 & 6.16 & 0.13 & 0.13 & 0.13 & 6.12 & 5.87 & 6.00 \\
\hline $\mathrm{N}_{1} \mathrm{P}_{2}$ & 6.47 & 5.69 & 6.08 & 0.15 & 0.14 & 0.14 & 6.11 & 5.85 & 5.98 \\
\hline $\mathrm{N}_{2} \mathrm{P}_{1}$ & 6.42 & 5.80 & 6.11 & 0.13 & 0.13 & 0.13 & 6.05 & 5.82 & 5.94 \\
\hline $\mathrm{N}_{2} \mathrm{P}_{2}$ & 6.45 & 5.73 & 6.09 & 0.14 & 0.14 & 0.14 & 6.05 & 5.82 & 5.94 \\
\hline $\mathrm{N}_{3} \mathrm{P}_{1}$ & 6.43 & 5.79 & 6.11 & 0.13 & 0.14 & 0.13 & 6.04 & 5.79 & 5.92 \\
\hline $\mathrm{N}_{3} \mathrm{P}_{2}$ & 6.49 & 5.78 & 6.13 & 0.12 & 0.12 & 0.12 & 6.06 & 5.83 & 5.94 \\
\hline S.Em. \pm & 0.02 & 0.03 & 0.02 & 0.006 & 0.005 & 0.004 & 0.009 & 0.011 & 0.007 \\
\hline C.D. at $5 \%$ & NS & NS & NS & NS & 0.01 & 0.01 & NS & NS & 0.021 \\
\hline $\mathrm{N}_{1} \mathrm{~K}_{1}$ & 6.44 & 5.71 & 6.07 & 0.13 & 0.13 & 0.13 & 6.13 & 5.89 & 6.01 \\
\hline $\mathrm{N}_{1} \mathrm{~K}_{2}$ & 6.43 & 5.84 & 6.13 & 0.14 & 0.14 & 0.14 & 6.12 & 5.85 & 5.99 \\
\hline $\mathrm{N}_{1} \mathrm{~K}_{3}$ & 6.57 & 5.74 & 6.15 & 0.14 & 0.13 & 0.14 & 6.09 & 5.83 & 5.96 \\
\hline $\mathrm{N}_{2} \mathrm{~K}_{1}$ & 6.42 & 5.87 & 6.14 & 0.13 & 0.13 & 0.13 & 6.06 & 5.84 & 5.95 \\
\hline $\mathrm{N}_{2} \mathrm{~K}_{2}$ & 6.48 & 5.71 & 6.10 & 0.13 & 0.15 & 0.14 & 6.05 & 5.82 & 5.94 \\
\hline $\mathrm{N}_{2} \mathrm{~K}_{3}$ & 6.42 & 5.72 & 6.07 & 0.13 & 0.13 & 0.13 & 6.04 & 5.80 & 5.92 \\
\hline $\mathrm{N}_{3} \mathrm{~K}_{1}$ & 6.48 & 5.85 & 6.16 & 0.14 & 0.14 & 0.14 & 6.06 & 5.83 & 5.94 \\
\hline $\mathrm{N}_{3} \mathrm{~K}_{2}$ & 6.42 & 5.78 & 6.10 & 0.12 & 0.13 & 0.13 & 6.04 & 5.80 & 5.92 \\
\hline $\mathrm{N}_{3} \mathrm{~K}_{3}$ & 6.48 & 5.71 & 6.09 & 0.12 & 0.13 & 0.12 & 6.05 & 5.81 & 5.93 \\
\hline S.Em. \pm & 0.03 & 0.04 & 0.03 & 0.008 & 0.006 & 0.004 & 0.011 & 0.014 & 0.009 \\
\hline C.D. at $5 \%$ & 0.08 & 0.12 & 0.08 & NS & NS & NS & NS & NS & NS \\
\hline $\mathrm{P}_{1} \mathrm{~K}_{1}$ & 6.44 & 5.83 & 6.14 & 0.13 & 0.13 & 0.13 & 6.09 & 5.86 & 5.97 \\
\hline $\mathrm{P}_{1} \mathrm{~K}_{2}$ & 6.41 & 5.83 & 6.12 & 0.13 & 0.14 & 0.13 & 6.06 & 5.81 & 5.94 \\
\hline $\mathrm{P}_{1} \mathrm{~K}_{3}$ & 6.49 & 5.75 & 6.12 & 0.12 & 0.12 & 0.12 & 6.06 & 5.81 & 5.94 \\
\hline $\mathrm{P}_{2} \mathrm{~K}_{1}$ & 6.45 & 5.78 & 6.12 & 0.14 & 0.13 & 0.14 & 6.08 & 5.85 & 5.96 \\
\hline $\mathrm{P}_{2} \mathrm{~K}_{2}$ & 6.47 & 5.73 & 6.10 & 0.13 & 0.13 & 0.13 & 6.08 & 5.83 & 5.96 \\
\hline $\mathrm{P}_{2} \mathrm{~K}_{3}$ & 6.48 & 5.69 & 6.09 & 0.14 & 0.13 & 0.14 & 6.06 & 5.81 & 5.94 \\
\hline S.Em. \pm & 0.02 & 0.03 & 0.02 & 0.006 & 0.005 & 0.004 & 0.009 & 0.011 & 0.007 \\
\hline C.D. at $5 \%$ & NS & NS & NS & NS & NS & NS & NS & NS & NS \\
\hline $\mathrm{N}_{1} \mathrm{P}_{1} \mathrm{~K}_{1}$ & 6.48 & 5.76 & 6.12 & 0.13 & 0.13 & 0.13 & 6.13 & 5.91 & 6.02 \\
\hline $\mathrm{N}_{1} \mathrm{P}_{1} \mathrm{~K}_{2}$ & 6.40 & 5.91 & 6.16 & 0.12 & 0.14 & 0.13 & 6.12 & 5.86 & 5.99 \\
\hline $\mathrm{N}_{1} \mathrm{P}_{1} \mathrm{~K}_{3}$ & 6.58 & 5.80 & 6.19 & 0.13 & 0.12 & 0.13 & 6.12 & 5.84 & 5.98 \\
\hline $\mathrm{N}_{1} \mathrm{P}_{2} \mathrm{~K}_{1}$ & 6.41 & 5.65 & 6.03 & 0.14 & 0.14 & 0.14 & 6.13 & 5.87 & 6.00 \\
\hline $\mathrm{N}_{1} \mathrm{P}_{2} \mathrm{~K}_{2}$ & 6.45 & 5.76 & 6.11 & 0.15 & 0.13 & 0.14 & 6.12 & 5.84 & 5.98 \\
\hline $\mathrm{N}_{1} \mathrm{P}_{2} \mathrm{~K}_{3}$ & 6.56 & 5.67 & 6.11 & 0.16 & 0.13 & 0.15 & 6.07 & 5.82 & 5.95 \\
\hline $\mathrm{N}_{2} \mathrm{P}_{1} \mathrm{~K}_{1}$ & 6.40 & 5.89 & 6.15 & 0.12 & 0.12 & 0.12 & 6.06 & 5.84 & 5.95 \\
\hline $\mathrm{N}_{2} \mathrm{P}_{1} \mathrm{~K}_{2}$ & 6.43 & 5.79 & 6.11 & 0.14 & 0.15 & 0.14 & 6.05 & 5.82 & 5.93 \\
\hline $\mathrm{N}_{2} \mathrm{P}_{1} \mathrm{~K}_{3}$ & 6.44 & 5.72 & 6.08 & 0.11 & 0.12 & 0.12 & 6.03 & 5.81 & 5.92 \\
\hline $\mathrm{N}_{2} \mathrm{P}_{2} \mathrm{~K}_{1}$ & 6.43 & 5.85 & 6.14 & 0.14 & 0.13 & 0.14 & 6.06 & 5.84 & 5.95 \\
\hline $\mathrm{N}_{2} \mathrm{P}_{2} \mathrm{~K}_{2}$ & 6.53 & 5.63 & 6.08 & 0.13 & 0.15 & 0.14 & 6.05 & 5.83 & 5.94 \\
\hline $\mathrm{N}_{2} \mathrm{P}_{2} \mathrm{~K}_{3}$ & 6.39 & 5.71 & 6.05 & 0.14 & 0.15 & 0.15 & 6.04 & 5.79 & 5.92 \\
\hline $\mathrm{N}_{3} \mathrm{P}_{1} \mathrm{~K}_{1}$ & 6.45 & 5.84 & 6.15 & 0.14 & 0.14 & 0.14 & 6.06 & 5.82 & 5.94 \\
\hline $\mathrm{N}_{3} \mathrm{P}_{1} \mathrm{~K}_{2}$ & 6.40 & 5.77 & 6.09 & 0.12 & 0.14 & 0.13 & 6.02 & 5.77 & 5.89 \\
\hline $\mathrm{N}_{3} \mathrm{P}_{1} \mathrm{~K}_{3}$ & 6.44 & 5.74 & 6.09 & 0.12 & 0.13 & 0.13 & 6.03 & 5.79 & 5.91 \\
\hline $\mathrm{N}_{3} \mathrm{P}_{2} \mathrm{~K}_{1}$ & 6.52 & 5.85 & 6.18 & 0.13 & 0.13 & 0.13 & 6.05 & 5.83 & 5.94 \\
\hline $\mathrm{N}_{3} \mathrm{P}_{2} \mathrm{~K}_{2}$ & 6.43 & 5.79 & 6.11 & 0.12 & 0.12 & 0.12 & 6.07 & 5.83 & 5.95 \\
\hline $\mathrm{N}_{3} \mathrm{P}_{2} \mathrm{~K}_{3}$ & 6.51 & 5.69 & 6.10 & 0.11 & 0.12 & 0.12 & 6.06 & 5.82 & 5.94 \\
\hline S.Em. \pm & 0.04 & 0.06 & 0.04 & 0.011 & 0.009 & 0.006 & 0.015 & 0.020 & 0.013 \\
\hline C.D. at $5 \%$ & NS & NS & NS & NS & NS & NS & NS & NS & NS \\
\hline Control & 6.44 & 5.60 & 6.02 & 0.16 & 0.13 & 0.15 & 6.15 & 5.87 & 5.79 \\
\hline S.Em. \pm & 0.04 & 0.06 & 0.04 & 0.011 & 0.009 & 0.006 & 0.015 & 0.021 & 0.013 \\
\hline C.D. at $5 \%$ & 0.11 & 0.18 & 0.11 & 0.03 & 0.02 & 0.02 & 0.044 & 0.059 & 0.037 \\
\hline
\end{tabular}

Note: FYM - $5 \mathrm{t} \mathrm{ha}^{-1} ; \mathrm{N}_{1}-100 \mathrm{~kg} \mathrm{ha}^{-1} ; \mathrm{N}_{2}-125 \mathrm{~kg} \mathrm{ha}^{-1} ; \mathrm{N}_{3^{-}} 150 \mathrm{~kg} \mathrm{ha}^{-1} ; \mathrm{P}_{1}-50 \mathrm{~kg} \mathrm{ha}^{-1} ; \mathrm{P}_{2}-75 \mathrm{~kg} \mathrm{ha}^{-1} ; \mathrm{K}_{1}-50 \mathrm{~kg} \mathrm{ha}^{-1} ; \mathrm{K}_{2}-75 \mathrm{~kg}$ $\mathrm{ha}^{-1} ; \mathrm{K}_{3}-100 \mathrm{~kg} \mathrm{ha}^{-1}$; NS - Non significant ; DAS - Days after sowing, Initial soil properties (2012): pH- 6.27; EC- $0.10 \mathrm{dS} \mathrm{m} \mathrm{m}^{-1}$; Organic carbon $-6.08 \mathrm{~g} \mathrm{~kg}^{-1}(2013)$ : $\mathrm{pH}-5.72$; EC- $0.11 \mathrm{dS} \mathrm{m}^{-1}$; Organic carbon - $5.88 \mathrm{~g} \mathrm{~kg}^{-1}$ 
T.V. Jyothi and N.S. Hebsur / J. Appl. \& Nat. Sci. 9 (4): 2315 - 2326 (2017)

Table 8. Available nutrients status as influenced by different levels of NPK at harvest of Bt cotton in Alfisol.

\begin{tabular}{|c|c|c|c|c|c|c|c|c|c|}
\hline \multirow{2}{*}{ Treatments } & \multicolumn{3}{|c|}{ Available nitrogen $\left(\mathrm{kg} \mathrm{ha}^{-1}\right)$} & \multicolumn{3}{|c|}{ Available phosphorus $\left(\mathbf{P}_{2} \mathrm{O}_{5}\right)\left(\mathrm{kgha}^{-1}\right)$} & \multicolumn{3}{|c|}{ Available potassium $\left(\mathrm{K}_{2} \mathrm{O}\right)\left(\mathrm{kg} \mathrm{ha}^{-1}\right)$} \\
\hline & 2012-13 & 2013-14 & Pooled & 2012-13 & 2013-14 & Pooled & 2012-13 & 2013-14 & Pooled \\
\hline $\mathrm{N}_{1}$ & 151.51 & 140.66 & 146.09 & 37.50 & 35.36 & 36.43 & 237.50 & 217.90 & 227.70 \\
\hline $\mathrm{N}_{2}$ & 146.61 & 137.89 & 142.25 & 35.09 & 33.24 & 34.17 & 233.64 & 214.28 & 223.96 \\
\hline $\mathrm{N}_{3}$ & 141.91 & 136.47 & 139.19 & 34.24 & 32.62 & 33.43 & 232.48 & 210.57 & 221.53 \\
\hline S.Em. \pm & 0.32 & 0.29 & 0.25 & 0.15 & 0.19 & 0.10 & 0.27 & 0.56 & 0.30 \\
\hline C.D. at $5 \%$ & 0.93 & 0.82 & 0.73 & 0.43 & 0.55 & 0.30 & 0.76 & 1.61 & 0.86 \\
\hline $\mathrm{P}_{1}$ & 147.59 & 138.50 & 143.05 & 35.98 & 33.90 & 34.94 & 234.67 & 214.86 & 224.77 \\
\hline $\mathrm{P}_{2}$ & 145.77 & 138.18 & 141.97 & 35.24 & 33.58 & 34.41 & 234.41 & 213.64 & 224.03 \\
\hline S.Em. \pm & 0.26 & 0.23 & 0.21 & 0.12 & 0.16 & 0.08 & 0.22 & 0.46 & 0.24 \\
\hline C.D. at $5 \%$ & 0.76 & NS & 0.60 & 0.35 & NS & 0.24 & NS & NS & 0.70 \\
\hline $\mathrm{K}_{1}$ & 150.14 & 140.62 & 145.38 & 36.59 & 34.74 & 35.66 & 235.88 & 217.00 & 226.44 \\
\hline $\mathrm{K}_{2}$ & 146.43 & 137.80 & 142.11 & 35.30 & 33.42 & 34.36 & 233.99 & 213.78 & 223.88 \\
\hline $\mathrm{K}_{3}$ & 143.48 & 136.60 & 140.04 & 34.93 & 33.06 & 34.00 & 233.76 & 211.97 & 222.87 \\
\hline S.Em. \pm & 0.32 & 0.29 & 0.25 & 0.15 & 0.19 & 0.10 & 0.27 & 0.56 & 0.30 \\
\hline C.D. at $5 \%$ & 0.93 & 0.82 & 0.73 & 0.43 & 0.55 & 0.30 & 0.76 & 1.61 & 0.86 \\
\hline $\mathrm{N}_{1} \mathrm{P}_{1}$ & 152.30 & 140.97 & 146.64 & 37.97 & 35.77 & 36.87 & 237.63 & 218.95 & 228.29 \\
\hline $\mathrm{N}_{1} \mathrm{P}_{2}$ & 150.72 & 140.36 & 145.54 & 37.03 & 34.95 & 35.99 & 237.37 & 216.85 & 227.11 \\
\hline $\mathrm{N}_{2} \mathrm{P}_{1}$ & 149.30 & 138.79 & 144.04 & 36.22 & 33.26 & 34.74 & 234.59 & 215.36 & 224.98 \\
\hline $\mathrm{N}_{2} \mathrm{P}_{2}$ & 143.92 & 137.00 & 140.46 & 33.96 & 33.22 & 33.59 & 232.70 & 213.20 & 222.95 \\
\hline $\mathrm{N}_{3} \mathrm{P}_{1}$ & 141.17 & 135.75 & 138.46 & 33.74 & 32.67 & 33.20 & 231.80 & 210.26 & 221.03 \\
\hline $\mathrm{N}_{3} \mathrm{P}_{2}$ & 142.66 & 137.19 & 139.92 & 34.73 & 32.57 & 33.65 & 233.17 & 210.88 & 222.03 \\
\hline S.Em. \pm & 0.46 & 0.40 & 0.36 & 0.21 & 0.27 & 0.15 & 0.38 & 0.79 & 0.42 \\
\hline C.D. at $5 \%$ & 1.32 & 1.16 & 1.04 & 0.60 & NS & 0.42 & 1.08 & NS & 1.22 \\
\hline $\mathrm{N}_{1} \mathrm{~K}_{1}$ & 156.14 & 143.03 & 149.58 & 38.68 & 36.51 & 37.60 & 238.61 & 221.00 & 229.81 \\
\hline $\mathrm{N}_{1} \mathrm{~K}_{2}$ & 151.47 & 140.92 & 146.19 & 37.30 & 35.45 & 36.37 & 237.47 & 218.10 & 227.78 \\
\hline $\mathrm{N}_{1} \mathrm{~K}_{3}$ & 146.94 & 138.05 & 142.49 & 36.54 & 34.11 & 35.32 & 236.42 & 214.59 & 225.51 \\
\hline $\mathrm{N}_{2} \mathrm{~K}_{1}$ & 150.59 & 140.13 & 145.36 & 35.89 & 34.31 & 35.10 & 235.09 & 217.31 & 226.20 \\
\hline $\mathrm{N}_{2} \mathrm{~K}_{2}$ & 147.48 & 137.91 & 142.69 & 35.03 & 33.23 & 34.13 & 233.91 & 214.74 & 224.32 \\
\hline $\mathrm{N}_{2} \mathrm{~K}_{3}$ & 141.77 & 135.63 & 138.70 & 34.35 & 32.19 & 33.27 & 231.94 & 210.80 & 221.37 \\
\hline $\mathrm{N}_{3} \mathrm{~K}_{1}$ & 143.69 & 138.72 & 141.20 & 35.21 & 33.39 & 34.30 & 233.93 & 212.69 & 223.31 \\
\hline $\mathrm{N}_{3} \mathrm{~K}_{2}$ & 140.33 & 134.57 & 137.45 & 33.59 & 31.59 & 32.59 & 230.59 & 208.52 & 219.55 \\
\hline $\mathrm{N}_{3} \mathrm{~K}_{3}$ & 141.72 & 136.12 & 138.92 & 33.91 & 32.89 & 33.40 & 232.93 & 210.52 & 221.73 \\
\hline S.Em. \pm & 0.56 & 0.50 & 0.44 & 0.26 & 0.33 & 0.18 & 0.46 & 0.97 & 0.52 \\
\hline C.D. at $5 \%$ & 1.61 & 1.42 & 1.27 & NS & 0.95 & 0.52 & 1.32 & 2.78 & 1.49 \\
\hline $\mathrm{P}_{1} \mathrm{~K}_{1}$ & 152.05 & 141.35 & 146.70 & 37.00 & 34.91 & 35.96 & 236.20 & 218.14 & 227.17 \\
\hline $\mathrm{P}_{1} \mathrm{~K}_{2}$ & 146.70 & 137.33 & 142.01 & 35.44 & 33.28 & 34.36 & 233.96 & 213.61 & 223.78 \\
\hline $\mathrm{P}_{1} \mathrm{~K}_{3}$ & 144.02 & 136.83 & 140.43 & 35.49 & 33.51 & 34.50 & 233.86 & 212.82 & 223.34 \\
\hline $\mathrm{P}_{2} \mathrm{~K}_{1}$ & 148.22 & 139.90 & 144.06 & 36.19 & 34.56 & 35.37 & 235.55 & 215.85 & 225.70 \\
\hline $\mathrm{P}_{2} \mathrm{~K}_{2}$ & 146.15 & 138.27 & 142.21 & 35.17 & 33.56 & 34.37 & 234.02 & 213.96 & 223.99 \\
\hline $\mathrm{P}_{2} \mathrm{~K}_{3}$ & 142.93 & 136.37 & 139.65 & 34.37 & 32.62 & 33.49 & 233.67 & 211.12 & 222.39 \\
\hline S.Em. \pm & 0.46 & 0.40 & 0.36 & 0.21 & 0.27 & 0.15 & 0.38 & 0.79 & 0.42 \\
\hline C.D. at $5 \%$ & 1.32 & 1.16 & 1.04 & NS & NS & 0.42 & NS & NS & NS \\
\hline $\mathrm{N}_{1} \mathrm{P}_{1} \mathrm{~K}_{1}$ & 157.28 & 143.50 & 150.39 & 38.73 & 37.22 & 37.98 & 239.10 & 222.88 & 230.99 \\
\hline $\mathrm{N}_{1} \mathrm{P}_{1} \mathrm{~K}_{2}$ & 152.49 & 140.86 & 146.67 & 37.68 & 35.59 & 36.63 & 237.57 & 218.41 & 227.99 \\
\hline $\mathrm{N}_{1} \mathrm{P}_{1} \mathrm{~K}_{3}$ & 147.15 & 138.55 & 142.85 & 37.51 & 34.51 & 36.01 & 236.22 & 215.55 & 225.89 \\
\hline $\mathrm{N}_{1} \mathrm{P}_{2} \mathrm{~K}_{1}$ & 154.99 & 142.55 & 148.77 & 38.62 & 35.81 & 37.22 & 238.13 & 219.12 & 228.63 \\
\hline $\mathrm{N}_{1} \mathrm{P}_{2} \mathrm{~K}_{2}$ & 150.45 & 140.98 & 145.71 & 36.91 & 35.32 & 36.12 & 237.36 & 217.78 & 227.57 \\
\hline $\mathrm{N}_{1} \mathrm{P}_{2} \mathrm{~K}_{3}$ & 146.74 & 137.54 & 142.14 & 35.56 & 33.71 & 34.64 & 236.61 & 213.64 & 225.13 \\
\hline $\mathrm{N}_{2} \mathrm{P}_{1} \mathrm{~K}_{1}$ & 153.46 & 140.96 & 147.21 & 36.92 & 34.18 & 35.55 & 235.60 & 217.70 & 226.65 \\
\hline $\mathrm{N}_{2} \mathrm{P}_{1} \mathrm{~K}_{2}$ & 150.05 & 138.83 & 144.44 & 36.27 & 33.33 & 34.80 & 235.14 & 216.30 & 225.72 \\
\hline $\mathrm{N}_{2} \mathrm{P}_{1} \mathrm{~K}_{3}$ & 144.40 & 136.57 & 140.49 & 35.46 & 32.27 & 33.86 & 233.02 & 212.09 & 222.56 \\
\hline $\mathrm{N}_{2} \mathrm{P}_{2} \mathrm{~K}_{1}$ & 147.72 & 139.31 & 143.51 & 34.86 & 34.43 & 34.65 & 234.57 & 216.92 & 225.75 \\
\hline $\mathrm{N}_{2} \mathrm{P}_{2} \mathrm{~K}_{2}$ & 144.90 & 136.99 & 140.95 & 33.78 & 33.12 & 33.45 & 232.67 & 213.17 & 222.92 \\
\hline $\mathrm{N}_{2} \mathrm{P}_{2} \mathrm{~K}_{3}$ & 139.14 & 134.69 & 136.92 & 33.24 & 32.11 & 32.68 & 230.85 & 209.51 & 220.18 \\
\hline $\mathrm{N}_{3} \mathrm{P}_{1} \mathrm{~K}_{1}$ & 145.42 & 139.59 & 142.51 & 35.35 & 33.34 & 34.34 & 233.92 & 213.85 & 223.89 \\
\hline $\mathrm{N}_{3} \mathrm{P}_{1} \mathrm{~K}_{2}$ & 137.56 & 132.29 & 134.92 & 32.36 & 30.93 & 31.65 & 229.15 & 206.11 & 217.63 \\
\hline $\mathrm{N}_{3} \mathrm{P}_{1} \mathrm{~K}_{3}$ & 140.52 & 135.37 & 137.95 & 33.51 & 33.74 & 33.62 & 232.32 & 210.83 & 221.58 \\
\hline $\mathrm{N}_{3} \mathrm{P}_{2} \mathrm{~K}_{1}$ & 141.96 & 137.84 & 139.90 & 35.08 & 33.44 & 34.26 & 233.94 & 211.52 & 222.73 \\
\hline $\mathrm{N}_{3} \mathrm{P}_{2} \mathrm{~K}_{2}$ & 143.11 & 136.85 & 139.98 & 34.82 & 32.25 & 33.53 & 232.03 & 210.92 & 221.47 \\
\hline $\mathrm{N}_{3} \mathrm{P}_{2} \mathrm{~K}_{3}$ & 142.91 & 136.88 & 139.89 & 34.30 & 32.04 & 33.17 & 233.54 & 210.21 & 221.88 \\
\hline S.Em.土 & 0.79 & 0.70 & 0.62 & 0.36 & 0.47 & 0.25 & 0.65 & 1.37 & 0.73 \\
\hline C.D. at $5 \%$ & 2.28 & 2.01 & 1.80 & 1.04 & 1.34 & 0.73 & 1.87 & 3.94 & 2.11 \\
\hline Control & 128.04 & 115.67 & 121.86 & 25.54 & 20.55 & 23.05 & 210.33 & 177.60 & 193.97 \\
\hline S.Em. \pm & 0.79 & 0.68 & 0.61 & 0.37 & 0.47 & 0.27 & 0.68 & 1.34 & 0.73 \\
\hline C.D. at $5 \%$ & 2.25 & 1.96 & 1.76 & 1.07 & 1.35 & 0.79 & 1.94 & 3.86 & 2.11 \\
\hline
\end{tabular}

Note: FYM - $5 \mathrm{t} \mathrm{ha}^{-1} ; \mathrm{N}_{1}-100 \mathrm{~kg} \mathrm{ha}^{-1} ; \mathrm{N}_{2}-125 \mathrm{~kg} \mathrm{ha}^{-1} ; \mathrm{N}_{3^{-}} 150 \mathrm{~kg} \mathrm{ha}^{-1} ; \mathrm{P}_{1}-50 \mathrm{~kg} \mathrm{ha}^{-1} ; \mathrm{P}_{2}-75 \mathrm{~kg} \mathrm{ha}^{-1} ; \mathrm{K}_{1}-50 \mathrm{~kg} \mathrm{ha}^{-1} ; \mathrm{K}_{2}-75 \mathrm{~kg} \mathrm{ha}^{-1} ; \mathrm{K}_{3}$ - $100 \mathrm{~kg} \mathrm{ha}^{-1}$; NS - Non significant ; DAS - Days after sowing, Initial soil nutrients status (2012) :Available nitrogen - $131.40 \mathrm{~kg}^{-1}$, Available $\mathrm{P}_{2} \mathrm{O}_{5}-28.60 \mathrm{~kg} \mathrm{ha}^{-1}$; Available $\mathrm{K}_{2} \mathrm{O}-208.90 \mathrm{~kg} \mathrm{ha}^{-1}$, (2013) :Available nitrogen - 119.26 kg ha ${ }^{-1}$; Available $\mathrm{P}_{2} \mathrm{O}_{5}-25.40 \mathrm{~kg}^{-1} \mathrm{ha}^{-1}$; Available $\mathrm{K}_{2} \mathrm{O}-181.22 \mathrm{~kg} \mathrm{ha}^{-1}$. 
T.V. Jyothi and N.S. Hebsur / J. Appl. \& Nat. Sci. 9 (4): 2315 - 2326 (2017)

Table 9. DTPA extractable micronutrients status as influenced by different levels of NPK at harvest of Bt cotton in Alfisol.

\begin{tabular}{|c|c|c|c|c|c|c|c|c|c|c|c|c|}
\hline \multirow{3}{*}{ Treatments } & \multicolumn{12}{|c|}{ DTPA extractable micronutrients $\left(\mathrm{mg} \mathrm{kg}^{-1}\right)$} \\
\hline & \multicolumn{3}{|c|}{ Zinc } & \multicolumn{3}{|c|}{ Iron } & \multicolumn{3}{|c|}{ Manganese } & \multicolumn{3}{|c|}{ Copper } \\
\hline & 2012-13 & 2013-14 & Pooled & $2012-13$ & 2013-14 & Pooled & $2012-13$ & 2013-14 & Pooled & $2012-13$ & 2013-14 & Pooled \\
\hline $\mathrm{N}_{1}$ & 0.53 & 0.53 & 0.53 & 4.61 & 7.59 & 6.10 & 9.23 & 14.40 & 11.81 & 1.25 & 1.22 & 1.24 \\
\hline $\mathrm{N}_{2}$ & 0.52 & 0.52 & 0.52 & 4.38 & 7.47 & 5.92 & 9.21 & 14.09 & 11.65 & 1.22 & 1.22 & 1.22 \\
\hline $\mathrm{N}_{3}$ & 0.52 & 0.52 & 0.52 & 4.18 & 7.42 & 5.80 & 9.21 & 14.16 & 11.69 & 1.22 & 1.20 & 1.21 \\
\hline S.Em. \pm & 0.003 & 0.002 & 0.002 & 0.07 & 0.05 & 0.05 & 0.006 & 0.14 & 0.07 & 0.006 & 0.008 & 0.006 \\
\hline C.D. at $5 \%$ & NS & NS & NS & 0.21 & NS & 0.13 & NS & NS & NS & 0.016 & NS & 0.017 \\
\hline $\mathrm{P}_{1}$ & 0.53 & 0.52 & 0.52 & 4.45 & 7.50 & 5.97 & 9.21 & 14.15 & 11.68 & 1.23 & 1.22 & 1.23 \\
\hline $\mathrm{P}_{2}$ & 0.52 & 0.52 & 0.52 & 4.33 & 7.49 & 5.91 & 9.22 & 14.28 & 11.75 & 1.23 & 1.21 & 1.22 \\
\hline S.Em. \pm & 0.002 & 0.002 & 0.002 & 0.06 & 0.04 & 0.04 & 0.005 & 0.11 & 0.06 & 0.005 & 0.006 & 0.005 \\
\hline C.D. at $5 \%$ & NS & NS & NS & NS & NS & NS & NS & NS & NS & NS & NS & NS \\
\hline $\mathrm{K}_{1}$ & 0.54 & 0.53 & 0.53 & 4.48 & 7.55 & 6.02 & 9.24 & 14.53 & 11.88 & 1.25 & 1.24 & 1.24 \\
\hline $\mathrm{K}_{2}$ & 0.52 & 0.52 & 0.52 & 4.36 & 7.47 & 5.91 & 9.21 & 14.16 & 11.69 & 1.23 & 1.21 & 1.22 \\
\hline $\mathrm{K}_{3}$ & 0.52 & 0.51 & 0.52 & 4.32 & 7.46 & 5.89 & 9.20 & 14.00 & 11.59 & 1.21 & 1.20 & 1.21 \\
\hline S.Em. \pm & 0.003 & 0.002 & 0.002 & 0.07 & 0.05 & 0.05 & 0.006 & 0.14 & 0.07 & 0.006 & 0.008 & 0.006 \\
\hline C.D. at $5 \%$ & 0.008 & 0.007 & 0.006 & NS & NS & NS & 0.018 & 0.40 & 0.20 & 0.016 & 0.022 & 0.017 \\
\hline $\mathrm{N}_{1} \mathrm{P}_{1}$ & 0.53 & 0.53 & 0.53 & 4.69 & 7.61 & 6.15 & 9.23 & 14.45 & 11.84 & 1.26 & 1.23 & 1.24 \\
\hline $\mathrm{N}_{1} \mathrm{P}_{2}$ & 0.52 & 0.52 & 0.52 & 4.52 & 7.56 & 6.04 & 9.22 & 14.36 & 11.79 & 1.25 & 1.22 & 1.23 \\
\hline $\mathrm{N}_{2} \mathrm{P}_{1}$ & 0.52 & 0.52 & 0.52 & 4.48 & 7.48 & 5.98 & 9.22 & 14.21 & 11.71 & 1.23 & 1.23 & 1.23 \\
\hline $\mathrm{N}_{2} \mathrm{P}_{2}$ & 0.53 & 0.52 & 0.52 & 4.28 & 7.45 & 5.86 & 9.21 & 13.96 & 11.59 & 1.21 & 1.21 & 1.21 \\
\hline $\mathrm{N}_{3} \mathrm{P}_{1}$ & 0.52 & 0.51 & 0.52 & 4.18 & 7.40 & 5.79 & 9.20 & 13.81 & 11.50 & 1.20 & 1.20 & 1.20 \\
\hline $\mathrm{N}_{3} \mathrm{P}_{2}$ & 0.52 & 0.53 & 0.53 & 4.18 & 7.45 & 5.81 & 9.23 & 14.52 & 11.88 & 1.23 & 1.21 & 1.22 \\
\hline S.Em. \pm & 0.004 & 0.003 & 0.003 & 0.11 & 0.07 & 0.06 & 0.009 & 0.20 & 0.10 & 0.008 & 0.011 & 0.008 \\
\hline C.D. at $5 \%$ & NS & 0.010 & 0.008 & NS & NS & NS & 0.025 & 0.56 & 0.29 & 0.023 & NS & NS \\
\hline $\mathrm{N}_{1} \mathrm{~K}_{1}$ & 0.53 & 0.54 & 0.54 & 4.71 & 7.65 & 6.18 & 9.25 & 14.61 & 11.93 & 1.27 & 1.24 & 1.25 \\
\hline $\mathrm{N}_{1} \mathrm{~K}_{2}$ & 0.53 & 0.52 & 0.53 & 4.58 & 7.57 & 6.08 & 9.23 & 14.45 & 11.84 & 1.26 & 1.23 & 1.24 \\
\hline $\mathrm{N}_{1} \mathrm{~K}_{3}$ & 0.52 & 0.51 & 0.52 & 4.53 & 7.54 & 6.03 & 9.20 & 14.14 & 11.67 & 1.23 & 1.21 & 1.22 \\
\hline $\mathrm{N}_{2} \mathrm{~K}_{1}$ & 0.54 & 0.53 & 0.53 & 4.50 & 7.51 & 6.01 & 9.24 & 14.50 & 11.87 & 1.25 & 1.24 & 1.24 \\
\hline $\mathrm{N}_{2} \mathrm{~K}_{2}$ & 0.53 & 0.52 & 0.52 & 4.36 & 7.48 & 5.92 & 9.22 & 14.07 & 11.64 & 1.22 & 1.23 & 1.23 \\
\hline $\mathrm{N}_{2} \mathrm{~K}_{3}$ & 0.51 & 0.51 & 0.51 & 4.27 & 7.41 & 5.84 & 9.18 & 13.69 & 11.44 & 1.20 & 1.19 & 1.20 \\
\hline $\mathrm{N}_{3} \mathrm{~K}_{1}$ & 0.54 & 0.52 & 0.53 & 4.24 & 7.49 & 5.86 & 9.22 & 14.46 & 11.84 & 1.24 & 1.23 & 1.24 \\
\hline $\mathrm{N}_{3} \mathrm{~K}_{2}$ & 0.51 & 0.51 & 0.51 & 4.14 & 7.36 & 5.75 & 9.20 & 13.95 & 11.58 & 1.21 & 1.18 & 1.19 \\
\hline $\mathrm{N}_{3} \mathrm{~K}_{3}$ & 0.52 & 0.52 & 0.52 & 4.17 & 7.42 & 5.79 & 9.21 & 14.08 & 11.65 & 1.21 & 1.20 & 1.20 \\
\hline S.Em. \pm & 0.005 & 0.004 & 0.003 & 0.13 & 0.09 & 0.08 & 0.011 & 0.24 & 0.12 & 0.010 & 0.013 & 0.010 \\
\hline C.D. at $5 \%$ & 0.015 & 0.012 & 0.010 & NS & NS & NS & NS & NS & NS & NS & NS & NS \\
\hline $\mathrm{P}_{1} \mathrm{~K}_{1}$ & 0.54 & 0.53 & 0.53 & 4.55 & 7.56 & 6.06 & 9.24 & 14.49 & 11.86 & 1.25 & 1.24 & 1.25 \\
\hline $\mathrm{P}_{1} \mathrm{~K}_{2}$ & 0.52 & 0.51 & 0.51 & 4.41 & 7.47 & 5.94 & 9.21 & 14.03 & 11.62 & 1.23 & 1.21 & 1.22 \\
\hline $\mathrm{P}_{1} \mathrm{~K}_{3}$ & 0.52 & 0.52 & 0.52 & 4.38 & 7.46 & 5.92 & 9.20 & 13.94 & 11.57 & 1.22 & 1.21 & 1.21 \\
\hline $\mathrm{P}_{2} \mathrm{~K}_{1}$ & 0.53 & 0.53 & 0.53 & 4.42 & 7.54 & 5.98 & 9.24 & 14.56 & 11.90 & 1.25 & 1.23 & 1.24 \\
\hline $\mathrm{P}_{2} \mathrm{~K}_{2}$ & 0.53 & 0.52 & 0.53 & 4.30 & 7.47 & 5.89 & 9.22 & 14.28 & 11.75 & 1.23 & 1.21 & 1.22 \\
\hline $\mathrm{P}_{2} \mathrm{~K}_{3}$ & 0.52 & 0.51 & 0.51 & 4.26 & 7.45 & 5.86 & 9.20 & 14.00 & 11.60 & 1.21 & 1.19 & 1.20 \\
\hline S.Em. \pm & 0.004 & 0.003 & 0.003 & 0.11 & 0.07 & 0.08 & 0.009 & 0.20 & 0.10 & 0.008 & 0.011 & 0.008 \\
\hline C.D. at $5 \%$ & NS & 0.010 & 0.008 & NS & NS & NS & NS & NS & NS & NS & NS & NS \\
\hline $\mathrm{N}_{1} \mathrm{P}_{1} \mathrm{~K}_{1}$ & 0.54 & 0.54 & 0.54 & 4.79 & 7.66 & 6.23 & 9.25 & 14.65 & 11.95 & 1.27 & 1.24 & 1.26 \\
\hline $\mathrm{N}_{1} \mathrm{P}_{1} \mathrm{~K}_{2}$ & 0.53 & 0.52 & 0.53 & 4.66 & 7.60 & 6.13 & 9.23 & 14.56 & 11.89 & 1.26 & 1.23 & 1.25 \\
\hline $\mathrm{N}_{1} \mathrm{P}_{1} \mathrm{~K}_{3}$ & 0.53 & 0.52 & 0.52 & 4.61 & 7.57 & 6.09 & 9.21 & 14.13 & 11.67 & 1.24 & 1.21 & 1.23 \\
\hline $\mathrm{N}_{1} \mathrm{P}_{2} \mathrm{~K}_{1}$ & 0.53 & 0.54 & 0.53 & 4.63 & 7.64 & 6.13 & 9.25 & 14.57 & 11.91 & 1.26 & 1.23 & 1.25 \\
\hline $\mathrm{N}_{1} \mathrm{P}_{2} \mathrm{~K}_{2}$ & 0.53 & 0.53 & 0.53 & 4.50 & 7.54 & 6.02 & 9.22 & 14.34 & 11.78 & 1.26 & 1.22 & 1.24 \\
\hline $\mathrm{N}_{1} \mathrm{P}_{2} \mathrm{~K}_{3}$ & 0.52 & 0.51 & 0.52 & 4.44 & 7.51 & 5.98 & 9.20 & 14.16 & 11.68 & 1.23 & 1.20 & 1.21 \\
\hline $\mathrm{N}_{2} \mathrm{P}_{1} \mathrm{~K}_{1}$ & 0.54 & 0.53 & 0.53 & 4.58 & 7.52 & 6.05 & 9.24 & 14.52 & 11.88 & 1.26 & 1.25 & 1.25 \\
\hline $\mathrm{N}_{2} \mathrm{P}_{1} \mathrm{~K}_{2}$ & 0.52 & 0.52 & 0.52 & 4.47 & 7.50 & 5.99 & 9.23 & 14.18 & 11.71 & 1.24 & 1.24 & 1.24 \\
\hline $\mathrm{N}_{2} \mathrm{P}_{1} \mathrm{~K}_{3}$ & 0.51 & 0.51 & 0.51 & 4.39 & 7.43 & 5.91 & 9.19 & 13.92 & 11.56 & 1.21 & 1.21 & 1.21 \\
\hline $\mathrm{N}_{2} \mathrm{P}_{2} \mathrm{~K}_{1}$ & 0.53 & 0.53 & 0.53 & 4.42 & 7.51 & 5.97 & 9.23 & 14.48 & 11.86 & 1.24 & 1.22 & 1.23 \\
\hline $\mathrm{N}_{2} \mathrm{P}_{2} \mathrm{~K}_{2}$ & 0.53 & 0.52 & 0.53 & 4.25 & 7.45 & 5.85 & 9.21 & 13.96 & 11.58 & 1.21 & 1.22 & 1.21 \\
\hline $\mathrm{N}_{2} \mathrm{P}_{2} \mathrm{~K}_{3}$ & 0.51 & 0.50 & 0.51 & 4.16 & 7.39 & 5.77 & 9.18 & 13.46 & 11.32 & 1.19 & 1.18 & 1.18 \\
\hline $\mathrm{N}_{3} \mathrm{P}_{1} \mathrm{~K}_{1}$ & 0.54 & 0.52 & 0.53 & 4.28 & 7.50 & 5.89 & 9.22 & 14.29 & 11.76 & 1.23 & 1.23 & 1.23 \\
\hline $\mathrm{N}_{3} \mathrm{P}_{1} \mathrm{~K}_{2}$ & 0.50 & 0.48 & 0.49 & 4.11 & 7.31 & 5.71 & 9.17 & 13.36 & 11.27 & 1.19 & 1.15 & 1.17 \\
\hline $\mathrm{N}_{3} \mathrm{P}_{1} \mathrm{~K}_{3}$ & 0.53 & 0.52 & 0.52 & 4.15 & 7.40 & 5.77 & 9.20 & 13.77 & 11.48 & 1.21 & 1.21 & 1.21 \\
\hline $\mathrm{N}_{3} \mathrm{P}_{2} \mathrm{~K}_{1}$ & 0.54 & 0.53 & 0.53 & 4.20 & 7.48 & 5.84 & 9.23 & 14.63 & 11.93 & 1.25 & 1.23 & 1.24 \\
\hline $\mathrm{N}_{3} \mathrm{P}_{2} \mathrm{~K}_{2}$ & 0.52 & 0.53 & 0.52 & 4.16 & 7.42 & 5.79 & 9.22 & 14.55 & 11.89 & 1.22 & 1.20 & 1.21 \\
\hline $\mathrm{N}_{3} \mathrm{P}_{2} \mathrm{~K}_{3}$ & 0.52 & 0.53 & 0.52 & 4.18 & 7.45 & 5.82 & 9.23 & 14.39 & 11.81 & 1.21 & 1.20 & 1.20 \\
\hline S.Em. \pm & 0.007 & 0.006 & 0.005 & 0.18 & 0.13 & 0.11 & 0.015 & 0.34 & 0.17 & 0.014 & 0.019 & 0.014 \\
\hline C.D. at $5 \%$ & NS & NS & NS & NS & NS & NS & NS & NS & NS & NS & NS & NS \\
\hline Control & 0.44 & 0.43 & 0.43 & 3.87 & 6.90 & 5.38 & 8.77 & 12.25 & 10.51 & 1.07 & 1.04 & 1.06 \\
\hline S.Em. \pm & 0.008 & 0.006 & 0.005 & 0.18 & 0.13 & 0.11 & 0.030 & 0.33 & 0.17 & 0.014 & 0.018 & 0.014 \\
\hline C.D. at $5 \%$ & 0.023 & 0.019 & 0.014 & 0.51 & 0.36 & 0.31 & 0.087 & 0.94 & 0.49 & 0.039 & 0.052 & 0.040 \\
\hline
\end{tabular}

Note: FYM - $5 \mathrm{t} \mathrm{ha}^{-1} ; \mathrm{N}_{1}-100 \mathrm{~kg} \mathrm{ha}^{-1} ; \mathrm{N}_{2}-125 \mathrm{~kg} \mathrm{ha}^{-1} ; \mathrm{N}_{3}-150 \mathrm{~kg} \mathrm{ha}^{-1} ; \mathrm{P}_{1}-50 \mathrm{~kg} \mathrm{ha}^{-1} ; \mathrm{P}_{2}-75 \mathrm{~kg} \mathrm{ha}^{-1} ; \mathrm{K}_{1}-50 \mathrm{~kg} \mathrm{ha}^{-1} ; \mathrm{K}_{2}-75 \mathrm{~kg} \mathrm{ha}^{-1} ; \mathrm{K}_{3}$ - $100 \mathrm{~kg} \mathrm{ha}^{-1}$; NS - Non significant ; DAS - Days after sowing, Initial soil nutrients status (2012):DTPA Zn- $0.59 \mathrm{mg} \mathrm{kg}^{-1}$; DTPA Fe - $4.91 \mathrm{mg}$ $\mathrm{kg}^{-1}$; DTPA Mn - $10.12 \mathrm{mg} \mathrm{kg}^{-1}$; DTPA Cu $-1.30 \mathrm{mg} \mathrm{kg}^{-1}$ (2013):DTPA Zn- $0.57 \mathrm{mg} \mathrm{kg}^{-1}$; DTPA Fe - $7.56 \mathrm{mg} \mathrm{kg}^{-1} ;$ DTPA Mn - $14.28 \mathrm{mg}$ $\mathrm{kg}^{-1} ;$ DTPA $\mathrm{Cu}-1.23 \mathrm{mg} \mathrm{kg}^{-1}$ 
pounds (ATP), which regulate photosynthesis. Gadhiya et al. (2009) revealed that among the three levels each of $\mathrm{N}\left(160,200\right.$ and $\left.240 \mathrm{~kg} \mathrm{ha}^{-1}\right)$, an application of $240 \mathrm{~kg} \mathrm{~N}^{-1}$ registered significantly higher values of $\mathrm{N}, \mathrm{P}$ and $\mathrm{K}$ uptake by cotton stalk on account of higher production of seed cotton yield and dry matter in medium black soils of Khedbhrahma (Gujarat). The positive response of K fertilizer @ $84 \mathrm{~kg} \mathrm{~K} \mathrm{ha}^{-1}$ on $\mathrm{K}$-deficient soils has been reported earlier by Reeves and Mullins (1995) in sandy loam soils of east-central Alabama. It is apparent that ability of soil to supply $\mathrm{K}^{+}$ to the roots is more important in determining $\mathrm{K}$ uptake than ability of roots to absorb $\mathrm{K}^{+}$from soil solution to meet total $\mathrm{K}$ requirement of the crop calculated on the basis of mass flow (Barber, 1985). Moreover, the application of the whole dose of $\mathrm{K}$ fertilizer at sowing time enhanced the soil's ability to increase $\mathrm{K}^{+}$concentration in the solution; favouring $\mathrm{N}$ uptake for vigorous vegetative growth particularly during early part of the season (Ali et al., 2007).

Effect of NPK fertilizers on fertility status of soil at harvest of Bt cotton in Alfisols: The available nitrogen, phosphorus and potassium in soil at harvest were significantly influenced by graded levels of NPK fertilizers but showed non significant effect on physicochemical properties of soil (pH, EC and OC) (Table 7). The treatment $\mathrm{N}_{1} \mathrm{P}_{1} \mathrm{~K}_{1}\left(100: 50: 50 \mathrm{~kg} \mathrm{~N}: \mathrm{P}_{2} \mathrm{O}_{5}: \mathrm{K}_{2} \mathrm{O}\right.$ ha $\left.{ }^{-1}\right)$ recorded significantly $(\mathrm{P}=0.05)$ higher available nitrogen $\left(150.39 \mathrm{~kg} \mathrm{ha}^{-1}\right)$, phosphorus (37.98 kg ha-1) and potassium (230.99 $\mathrm{kg} \mathrm{ha}^{-1}$ ) compared to other treatments. The application of 150:50:75 kg N:P $\mathrm{O}_{5}: \mathrm{K}_{2} \mathrm{O}$ $\mathrm{ha}^{-1}\left(\mathrm{~N}_{3} \mathrm{P}_{1} \mathrm{~K}_{2}\right)$ recorded lower available nitrogen (134.92 kg ha $\mathrm{kg}^{-1}$ ), phosphorus (31.65 kg ha ${ }^{-1}$ ) and potassium (217.63 $\mathrm{kg} \mathrm{ha}^{-1}$ ) (Table 8). The interaction effect of graded levels of NPK fertilizers remained non significant for DTPA extractable micronutrients $(\mathrm{Cu}, \mathrm{Fe}$, $\mathrm{Mn}$ and $\mathrm{Zn}$ ) (Table 9). The reason for lower available nutrients under these treatments might be due to the supply of these nutrients at higher levels and higher uptake by the crop.

\section{Conclusion}

From the experimental results it can be concluded that, the application of 150:50:75 kg N: $\mathrm{P}_{2} \mathrm{O}_{5}: \mathrm{K}_{2} \mathrm{O}$ ha $^{-1}$ $\left(\mathrm{N}_{3} \mathrm{P}_{1} \mathrm{~K}_{2}\right)$ recorded significantly higher nitrogen (132.63 kg ha $\left.{ }^{-1}\right)$, phosphorus ( $\left.31.26 \mathrm{~kg} \mathrm{ha}^{-1}\right)$ and potassium $\left(128.94 \mathrm{~kg} \mathrm{ha}^{-1}\right)$ uptake by cotton. The treatment receiving 100:50:50 kg N: $\mathrm{P}_{2} \mathrm{O}_{5}: \mathrm{K}_{2} \mathrm{O}$ ha ${ }^{-1}\left(\mathrm{~N}_{1} \mathrm{P}_{1} \mathrm{~K}_{1}\right)$ recorded significantly highest available nitrogen (150.39 $\mathrm{kg} \mathrm{ha}^{-1}$ ), available phosphorus (37.98 $\mathrm{kg} \mathrm{ha}^{-1}$ ) and available potassium (230.99 $\mathrm{kg} \mathrm{ha}^{-1}$ ) compared to rest of the treatments. The lowest available nitrogen (134.92 $\left.\mathrm{kg} \mathrm{ha}^{-1}\right)$, available phosphorus $\left(31.65 \mathrm{~kg} \mathrm{ha}^{-1}\right)$ and available potassium $\left(217.63 \mathrm{~kg} \mathrm{ha}^{-1}\right)$ were recorded in treatment receiving 150:50:75 kg $\mathrm{N}: \mathrm{P}_{2} \mathrm{O}_{5}: \mathrm{K}_{2} \mathrm{O}$ ha $^{-1}\left(\mathrm{~N}_{3} \mathrm{P}_{1} \mathrm{~K}_{2}\right)$. The superiority of 150:50:75 kg N: $\mathrm{P}_{2} \mathrm{O}_{5}: \mathrm{K}_{2} \mathrm{O}$ ha $^{-1}$ in improving uptake and soil properties over the other treatments except the one supplied with 125:75:100 kg N: $\mathrm{P}_{2} \mathrm{O}_{5}: \mathrm{K}_{2} \mathrm{O}$ ha $^{-1}$. Hence, both the treatments emphasizing that any of these two can be considered as optimum dose for Alfisols.

\section{REFERENCES}

Ali, Muhammad Anjum, Yousaf Hassan Tatla and Muhammad Aslam (2007). Response of cotton (Gossypium hirsutum L.) to potassium fertilization in an arid environment. J. Agri. Res. 45 (3): 191-198.

Barber, S. A. (1985). Potassium availability at the soil-root interface and factors influencing potassium uptake. In: Munson RD, ed. Potassium in Agriculture, Madison, Wisconsin, USA: American Society of Agronomy, pp. 309-324.

Gadhiya, S. S., Patel, B. B., Jadav, N. J., Pavaya, R. P., Patel, M. V. and Patel, V. R., 2009, Effect of different levels of nitrogen, phosphorus and potassium on growth, yield and quality of Bt cotton. The Asian J. Hort., 4 (1): 37-42.

Lindsay, W. L. and Norvell, W. A. (1978). Development of a DTPA-soil test for Zn, Fe, Mn and Cu. Soil Sci. Soc. American J. 42: 421-428.

Marschner, H. (1995). Mineral Nutrition of Higher Plants ( $2^{\text {nd }}$ ed. $)$, Academic Press, London.

Mullins, G.L. and Burmester, C.H. (2010). Relation of growth and development to mineral nutrition. pp. 97105. In: J.M. Stewart, D.M. Oosterhuis, J.M. Heitholt, and J.R. Mauney (eds.) Physiology of Cotton. Springer, New York. http://www.springerlink.com/index/ u88838767k45m032.pdf.

Peuke, A. D., Jeschke, W. and Hartung, W. (2002). Flows of elements, ions and abscissic acid in Ricinus communis and site of nitrate reduction under potassium limitation. J. Exper. Bot. 53: 241-250.

Reeves, D. W. and Mullins, G. L., 1995, Sub-soiling and potassium effects on water relations and yield of cotton. Agron. J., 87: 847-852.

Rochester, I. J., Constable, G. A., Oosterhuis, D. M. and Meredith Errington (2012). Nutritional requirements of cotton during flowering and fruiting, Published by CSIRO Plant Industry, Narrabri, Australia.

Sharawat, K. L. and Burford, J. R. (1982). Modification of alkaline permanganate method for assessing the availability of soil nitrogen in upland soils.Soil Sci.133:53-57.

Sparks (1996). Methods of soil analysis, Part -3: Chemical Methods, Soil Science Society of America, USA.

Tandon, H. L. S. (1998). Methods of Analysis of Soils, Plants, Waters, Fertilizers and Organic Manures, Fertilizer development and consultation organization, New Delhi, India, p. 204.

Tayade, A. S. and Dhoble, M. V. (2010). Effect of transgenic Bt-cotton hybrid, nutrient and pest management on seed cotton yield, nutrient uptake and status of available nutrient in the soil. Indian J. Fert. 6(8): 34-40.

Waikar, S. L., Dhamak, A. L., Meshram, N. A. and Patil, V. D. (2015). Effect of Speciality fertilizers on soil fertility, nutrient uptake, quality and productivity of cotton in Vertisol. IOSR J. Agric. Veter. Sci. 8(2): 76-79.

Zakaria M. S., Ashraf H. F. and Serag E. Y. (2009) Direct and residual effects of nitrogen fertilization, foliar application of potassium and plant growth retardant on Egyptian cotton growth, seed yield, seed viability and seedling vigor. Acta Eco. Sinica, 29: 116-123. 